\title{
Analysis of Flow Field Characteristics and Pressure Pulsation in Horizontal Axis Double-Runner Francis Turbine
}

\author{
Chunxia Yang * $\mathbb{C}^{\text {, Jiawei } W u, ~ D i n g e ~} \mathrm{Xu}$, Yuan Zheng, Xueyuan Hu and Zhe Long \\ College of Energy and Electrical Engineering, Hohai University, Nanjing 210098, China; \\ 201306020011@hhu.edu.cn (J.W.); xudinge@hhu.edu.cn (D.X.); zhengyuan@hhu.edu.cn (Y.Z.); \\ 201606010028@hhu.edu.cn (X.H.); longzhe@hhu.edu.cn (Z.L.) \\ * Correspondence: yangchunxia@hhu.edu.cn; Tel.: +86-15895941359
}

check for updates

Citation: Yang, C.; Wu, J.; Xu, D.; Zheng, Y.; Hu, X.; Long, Z. Analysis of Flow Field Characteristics and Pressure Pulsation in Horizontal Axis Double-Runner Francis Turbine. Water 2021, 13, 2671. https:// doi.org/10.3390/w13192671

Academic Editor: Arthur Mynett

Received: 1 September 2021

Accepted: 23 September 2021

Published: 27 September 2021

Publisher's Note: MDPI stays neutral with regard to jurisdictional claims in published maps and institutional affiliations.

Copyright: (c) 2021 by the authors. Licensee MDPI, Basel, Switzerland. This article is an open access article distributed under the terms and conditions of the Creative Commons Attribution (CC BY) license (https:// creativecommons.org/licenses/by/ $4.0 /)$.

\begin{abstract}
Horizontal axis double-runner Francis turbines have great advantages in the development of small hydropower plants, but the arrangement of double runners aggravates the complexity of the water flow between runners, and the mutual influence of the two runners cannot be ignored. In order to explore the relationship between the performance and the internal flow field and investigate the pressure pulsation characteristics of the double-runner Francis turbine, the steady and unsteady numerical analysis of the full flow channel of a prototype turbine was carried out based on the Realizable k-epsilon model and the polyhedral mesh method. The results show that the relationship between the average efficiency of the two runners and the flow difference between the runners is negatively correlated. As the flow rate difference between the runners on both sides increases, the average efficiency of the runners decreases. The draft tube flow of a horizontal-axis turbine has a profound effect on the flow field characteristics in the runner. When the working conditions change, the turning and converging timing of the mainstream at the outlet of the two runners will change. The movement of the mainstream promotes the change in location of the dead water zone. The existence of the vortex zone makes the pressure distribution at the outlet of the runner uneven, which is an important reason for the asymmetry of the flow in the runner. The analysis of pressure pulsation and its frequency spectrum shows that when the working conditions change, the low-frequency, strong pressure pulsation area on the surface of the guide vane will regularly migrate between the two runners, while the high-frequency pressure pulsation that occurs in the bladeless zone will dissipate in the runner. The doubling of the blade frequency on the pressure surface and back surface of the blades gradually attenuates with the increase of frequency. The pressure pulsation attenuation on the surface of the high-position blade conforms to the linear law, and the attenuation of the pressure pulsation on the surface of the low-position blade conforms to the exponential law. The research in this paper provides a certain reference value for revealing the flow field mechanism and pressure pulsation characteristics of the double-runner Francis turbine.
\end{abstract}

Keywords: horizontal axis francis turbine; double runner; numerical simulation; flow field; pressure pulsation

\section{Introduction}

Small hydropower units, which are widely used in rural and remote hilly areas [1], often face complex operating conditions in which the head and flow rate vary over a range, and they have a greater magnitude of pulsations in the velocity and pressure in the flow field of the turbine when the operating point is further away from the design point [2]. Horizontal Francis turbines are widely used in small hydropower stations because of their simple structure, low investment, and convenient operation and maintenance, and are also widely used in hilly areas [3]. However, the high efficiency zone of Francis turbines is relatively narrow, especially in river basins with large flow changes. If the units are forced to start up during the flow trough period, not only can the quality of power generation 
be unsatisfied, but the hydraulic vibration caused by deviation from the design operating conditions can also cause great damage to the units themselves. If the plant is shut down during the dry season, it will directly lose all the water resources, which will affect the economic benefits of the plant [4-6]. Some engineers have previously introduced solutions for horizontal axis double-runner Francis turbines. For example, in his paper, Robert $[5,7]$ detailed some of the advantages of the horizontal axis double-runner Francis turbine over the traditional horizontal axis single-runner and vertical axis turbine schemes, focusing on the following two points:

(1) The horizontal axis double-runner turbine can save the investment and construction cost of the hydropower station. Due to the symmetrical flow of the horizontal double runner, the water thrust can offset each other, which can reduce the manufacturing cost of the thrust bearings; manufacturing and processing the double-runner turbines with the same capacity, the cost is only several percent higher than that of the single runner; and the cavitation performance is good, which can save the construction cost of the workshop [8].

(2) The horizontal axis double-runner turbine can flexibly cope with large flow change. When the flow rate of a single runner is less than $40 \%$ of the rated flow rate, the efficiency of the turbine will be seriously reduced due to the deterioration of flow regime and hydraulic vibration, while a double runner allows the hydraulic turbine to operate in the efficient zone by closing the guide vane or barrel valve of one runner [9].

In view of the above two obvious advantages, the horizontal axis double-runner turbine has considerable application prospects in the development of small hydropower stations. However, the application of such units in the world is still few [10]. Firstly, due to the development of hydropower resources still being in the creep stage, the development of super hydropower facilities is still under construction, and the demand for small hydropower is not urgent [11,12]; secondly, the research on such types of hydropower turbines to provide effective demonstrations for engineering construction is few.

The efficiency of Francis turbines at low flow rates is influenced by the pressure pulsation downstream of the runner, hydraulic oscillation of the draft tube, and pressure pulsation of the full flow channel [13], so the operation stability of the hydraulic unit is an important subject in the field of hydropower, which makes the pressure pulsation analysis of flow channels of hydraulic turbines very important. For horizontal axis units, due to the asymmetric flow of water in the vertical direction of the single runner, the unstable flow in the flow channel can easily cause the unit to vibrate and affect the normal and stable operation of the unit. The pressure pulsation characteristic has become the basic index to measure the stability of the hydraulic unit [14]. The operating stability of the horizontal axis double-runner turbine can be investigated by analyzing and studying its pressure pulsation frequency spectrum characteristics [3].

In recent years, based on existing research results, scholars in the world have further carried out numerical simulation and experimental research on the pressure pulsation of hydraulic turbines. Based on numerical simulation and model tests, the stability and pressure pulsation of Francis turbines under overload, part load, and deep part load were studied by Maiwald [15]. The results showed that different runner structure designs can affect the pressure pulsation level in the unit. In the same year, Nennemann [16] used an automated computational fluid dynamics (CFD) method to predict the pressure pulsations in the draft tube under partial load and to separate the pressure signals into synchronous, asynchronous, and random pulsation. After that, in the study of Minakov [17,18], the flow structure at the outlet of Francis turbine runners was analyzed, and the influence of flow structures on the frequency and intensity of pressure pulsation in the transition process of the runner was given. The results showed that the calculation is in good agreement with the experimental data. To compare the difference in pressure pulsation characteristics between horizontal and vertical axis turbines, Chirag [19] researched both the horizontal and vertical Francis turbines, where the results showed that the non-synchronous pressure pulsation had less influence on the internal flow field of the horizontal turbine than the 
synchronous one. In the latest study, Favrel [20] investigated the influence of the geometry of draft tubes of the Francis turbine on the pressure pulsation and energy loss under off-design conditions, and the results fully showed that the draft tube shape significantly affects the pressure pulsation distribution in the runner channel of the turbine. To some extent, both experimental and numerical methods reveal that the channel structure can highly affect the spatial-temporal distribution and frequency characteristics of the pressure pulsation of the turbine. Compared with the experimental method, it is difficult to capture the details of the flow regime, and the cost of the high-precision device is higher. In this case, the numerical calculation becomes prominent. Whether the numerical method is reliable or not requires more numerical methods to verify.

$\mathrm{Wu}$ [21] predicted and analyzed the amplitude and frequency of pressure pulsation inside the Kaplan turbine, and the results showed that the essence of the turbine system resonance was caused by the sudden increase of pressure pulsation at the inlet section of the draft tube. Liu et al. [22,23] combined the unsteady flow numerical simulation and model test method, and analyzed and proved that the rotor-stator interference between runner and guide vanes of Francis turbines and draft tube vortex ropes are the two main pulsating sources of pressure pulsation, and can propagate in the whole flow field of turbines. Feng et al. [24] studied the pressure pulsation characteristics of Francis turbines under off-design conditions, and concluded that the amplitude of pressure pulsation can be reduced by lengthening the runner cone, though the frequency cannot be changed. Ji et al. [25] obtained the propagation law of pressure pulsation in the runner through threedimensional unsteady turbulence simulation. The results showed that the low-frequency pulsation on the blade surface gradually attenuates from the blade outlet to the inlet, while the high-frequency pulsation has the opposite law. Zheng [26] carried out a numerical study on pressure pulsation of tubular turbines, and revealed the mechanism of lowfrequency pressure pulsation of tubular turbines under different conditions, indicating that the low-frequency pressure pulsation inside the turbine is caused by the vortex rope of the draft tube. By numerical and experimental methods, Su et al. [27] analyzed the unsteady characteristics of Francis turbine draft tubes, and found that the geometric design of the draft tube had a great influence on the pressure pulsation characteristics of the draft tube. Xie, Yang $[28,29]$ et al. studied the pressure pulsation characteristics of a dual-turbine system. The results showed that the unbalanced flow distribution in the dual-turbine system leads to a change in the location of the pressure pulsation. Some scholars also studied the mechanism of turbine pressure pulsation and its interaction with the flow field on the basis of improving the precision and exactitude of numerical calculation. In a study by Li [30], four turbulence models were used to simulate the pressure pulsation of Francis turbines. The results showed that the turbulence model is not monotonously linear to the mesh scale. Qian [13,31] compared the superiority-inferiority of three different methods for predicting the internal flow of hydraulic turbines, namely, large eddy simulation, Standard $k-\varepsilon$ and Realizable $k-\varepsilon$. The results showed that the Realizable $k-\varepsilon$ model can qualitatively analyze the pressure pulsation characteristics of the whole flow channel in the turbine, while the Standard $k-\varepsilon$ model has a larger error.

The double-runner unit has its own special structural form. Probing into its internal pressure pulsation characteristics is of significance to improve its applicability and application scope [32]. In this paper, on the basis of the research work carried out by the above scholars, based on the horizontal axis double-runner hydraulic unit which has been put into operation in a power plant in northern Europe, the three-dimensional solid model of the whole flow channel is established, and the three-dimensional steady and unsteady turbulent flow is calculated and analyzed by the CFD numerical calculation method. Through the comprehensive analysis of the hydraulic performance, the distribution characteristics of the internal flow field reveal the specific reasons for the change in hydraulic performance. Finally, a spectrum analysis of the pressure pulsation monitoring data is carried out to quantitatively analyze the hydraulic stability of such units. 


\section{Numerical Simulation Method}

\subsection{Geometric Model of Horizontal Axis Francis Double-Runner Turbine}

In this paper, a three-dimensional model of a horizontal axis double-runner prototype Francis turbine is adopted, and its full flow channel is shown in Figure 1. The turbine connects two runners through a main shaft, each with a mirror symmetrical runner arranged upstream (runner I) and downstream (runner II). The diameter of the runner is $D_{r}=1.7 \mathrm{~m}$, there is no spiral case and fixed guide vanes, the number of active guide vanes is $Z_{0}=20$, the number of runner vanes is $Z=17$, the rated speed is $n_{r}=250 \mathrm{r} \cdot \mathrm{min}^{-1}$, the rotating frequency of the runner is $f=4.16 \mathrm{~Hz}$, the rated power is $P_{r}=4500 \mathrm{~kW}$ under the rated head $H_{r}=25 \mathrm{~m}$, the runner outlet is arranged face-to-face, and two $90^{\circ}$ elbows converge into the straight cone section of the draft tube, in which the main shaft passes through the outlet elbow, and the axis of the draft tube diffusion section has an angle of $135^{\circ}$ with the turbine main shaft.

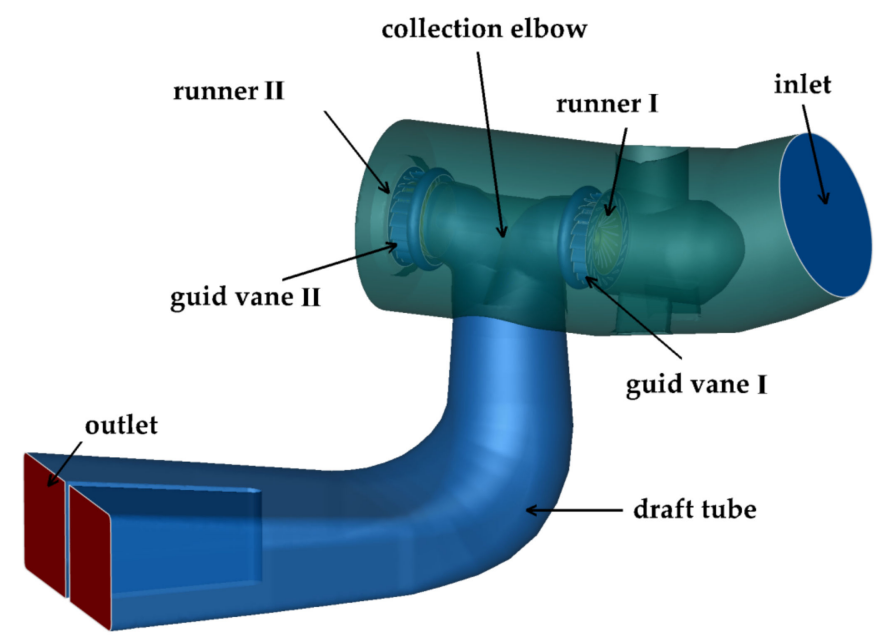

Figure 1. Full flow channel model of a double-runner Francis turbine.

\subsection{Computational Domain Discretization and Mesh Independence Validation}

Considering the complexity of the geometry structure, the octree algorithm with high robustness is used to draw unstructured tetrahedral mesh with good adaptability of the geometric surface. According to the research of $\mathrm{Xu}$ and Wei $[33,34]$, polyhedral mesh has the characteristics of being high quality, and having strong geometric adaptability and fast convergence, and the time consumed in the numerical calculation is only about $1 / 4-1 / 5$ of that in tetrahedral mesh. Considering the limitation of computing resources and to improve the mesh quality and computational efficiency, the computational domain is transformed into polyhedral mesh by using the built-in converter in the Fluent 2020 R2 solver. Through the mesh scale test, the calculated results of the transformed mesh are compared with those of the original tetrahedral mesh, which verifies that the polyhedral mesh can indeed play a good role in this model. The guide vane opening at $\mathrm{a}_{5}=110 \mathrm{~mm}$ (the Design Operation Condition) was adopted for mesh independence validation. Figure 2 shows the efficiency curves for different numbered schemes of tetrahedral and polyhedral mesh, where the $\mathrm{y}$-coordinate represents the average efficiency of two runners, which is the criterion of convergence of the calculation results. 


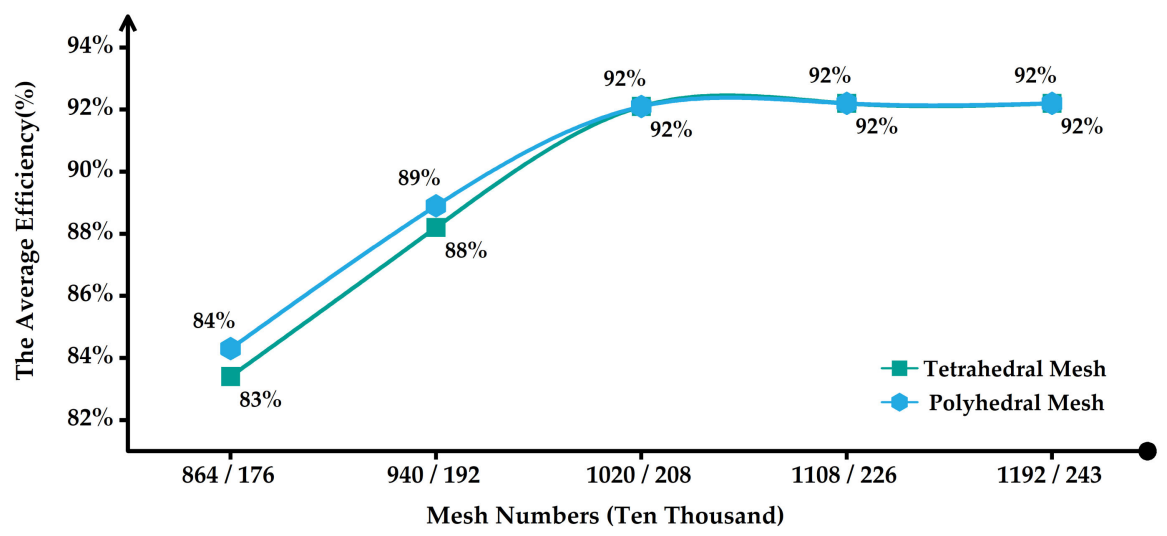

Figure 2. Variation curves of numerical calculation efficiency of different mesh schemes.

From the curves, we can see that the polyhedral mesh has the same results as the tetrahedral mesh. The computational process of mesh independence validation confirms its high computational efficiency and good convergence. Therefore, the polyhedral mesh with the quantity of 2.08 million was used for calculations. The $y+$ is 33 with this mesh. Figure 3 shows the mesh division diagram.

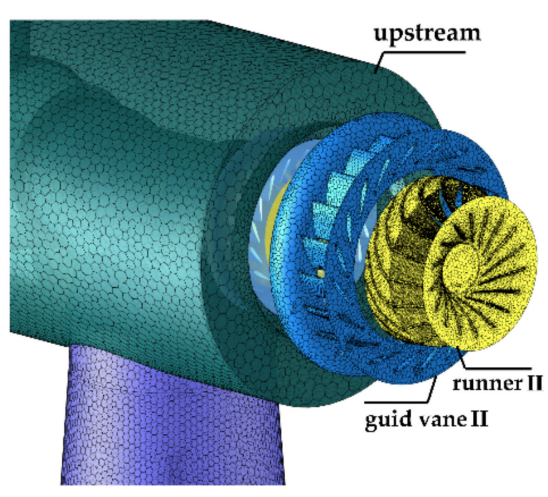

(a)

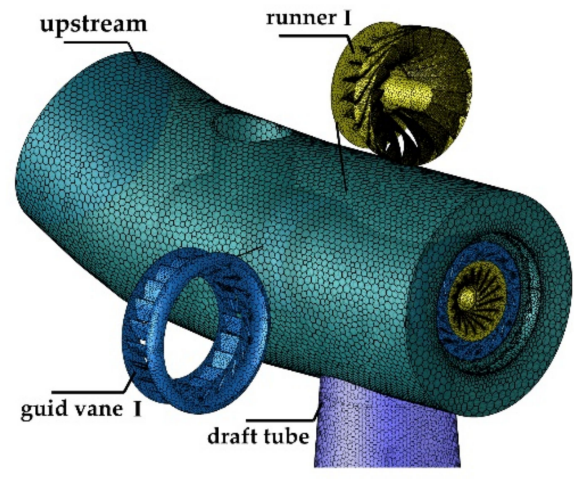

(b)

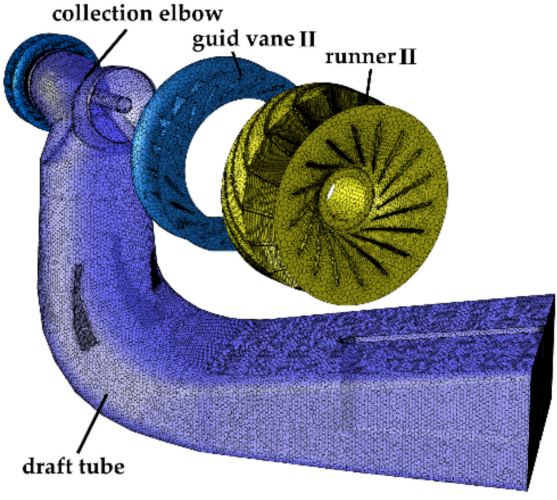

(c)

Figure 3. Diagram of mesh division. (a) Meshing of runner II guide vane II; (b) meshing of runner I guide vane I; (c) meshing of collection elbow and dtaft tube.

\subsection{Selection of Numerical Methods, Boundary Conditions and Calculation Conditions}

Different numerical models will have a significant impact on the accuracy of calculations, and the selection of an appropriate turbulence model can capture the required flow details more accurately [35]. The Standard $k-\varepsilon$ turbulence model may lead to negative normal stress when the time-averaged strain rate is particularly large. In order to make the flow conform to the physical law of turbulence, it is necessary to make some mathematical constraints on the normal stress. To ensure the realization of this constraint, Shih [36] believed that the coefficient $C_{\mu}$ should not be a constant, but should be related to the strain rate, so they put forward the Realizable $k-\varepsilon$ turbulence model, which is different from the Standard $k-\varepsilon$ model in two aspects: 1 . The realizable $k-\varepsilon$ model contains an alternative formulation for the turbulent viscosity. 2. A modified transport equation for the dissipation rate, $\varepsilon$, has been derived from an exact equation for the transport of the mean-square vorticity fluctuation [37]. The term "realizable" means that the model satisfies certain mathematical constraints on the Reynolds stresses, consistent with the physics of turbulent flows. Neither the standard $k-\varepsilon$ model nor the RNG $k-\varepsilon$ model is realizable. 
The transport equations of the model are as follows:

$$
\begin{gathered}
\frac{\partial}{\partial t}(\rho k)+\frac{\partial}{\partial x_{j}}\left(\rho k u_{j}\right)=\left[\left(\mu+\frac{\mu_{t}}{\sigma_{k}}\right) \frac{\partial k}{\partial x_{j}}\right]+G_{k}+G_{b}-\rho \varepsilon-Y_{M}+S_{k} \\
\frac{\partial}{\partial t}(\rho \varepsilon)+\frac{\partial}{\partial x_{j}}\left(\rho \varepsilon u_{j}\right)=\frac{\partial}{\partial x_{j}}\left[\left(\mu+\frac{\mu_{t}}{\sigma_{\varepsilon}}\right) \frac{\partial \varepsilon}{\partial x_{j}}\right]+\rho C_{1} S \varepsilon-\rho C_{2} \frac{\varepsilon^{2}}{k+\sqrt{v \varepsilon}}+C_{1 \varepsilon} \frac{\varepsilon}{k} C_{3 \varepsilon} G_{b}+S_{\varepsilon}
\end{gathered}
$$

where $k$ and $\varepsilon$ are the working variables of the transport equation, $G_{k}$ represents the generation of turbulence kinetic energy due to the mean velocity gradients, $G_{b}$ is the generation of turbulence kinetic energy due to buoyancy, $Y_{M}$ represents the contribution of the fluctuating dilatation in compressible turbulence to the overall dissipation rate, $C_{2}$ and $C_{1 \varepsilon}$ are constants, $\sigma_{k}$ and $\sigma_{\varepsilon}$ are the turbulent Prandtl numbers for $\mathrm{k}$ and $\varepsilon$, respectively, and $S_{k}$ and $S_{\varepsilon}$ are source terms defined in different cases. In the calculation, the constant values $C_{2}=1.9, C_{1 \varepsilon}=1.44, \sigma_{k}=1.0, \sigma_{\varepsilon}=1.2$ are defined.

As in other $k-\varepsilon$ models, the Eddy viscosity is computed from

$$
\mu_{t}=\rho C_{\mu} \frac{k^{2}}{\varepsilon}
$$

In the Realizable $k-\varepsilon$ model, $C_{\mu}$ is a variable defined by the following formula, and it is also a function of the mean strain and rotation rate, the angular velocity of the system rotation, and the turbulence fields $(k$ and $\varepsilon)$.

$$
C_{\mu}=\frac{1}{A_{0}+A_{S} \frac{k U^{*}}{\varepsilon}}
$$

The results show that the Realizable $k-\varepsilon$ model derived from the exact turbulence transport equation has the best performance in the validation of separated flows and flows with complex secondary flow characteristics [36]. In order to accurately simulate the fluid flow in the turbine and accurately capture the pressure pulsation information in the runner, the Realizable $k-\varepsilon$ model is selected based on the research of Qian [31].

The finite volume method is used to discretize the governing equations, the secondorder fully implicit scheme for unsteady terms, the central difference scheme for pressure and diffusion terms, and the second-order upwind scheme for convection terms are used to limit the numerical error and its propagation in flow. In the calculation model of the hydraulic turbine, all the structures are still, except the two runners which rotate at high speed. The Frozen Rotor method is used to set up the interface between the guide vane and draft tube and the runner. The wall is set to a no-slip wall, and the standard wall function is used to deal with the turbulent flow near the wall. A pressure inlet is set to deal with the boundary of the inlet, and the pressure is calculated according to the rated head of the hydraulic turbine. The total pressure $P=245,165 \mathrm{~Pa}$ and operation pressure $P_{o p}=101,325 \mathrm{~Pa}$. The outlet boundary condition is the pressure outlet, and the pressure value is set to 0 .

The uncertainties of the calculation are judged by checking the design parameters of the double-runner turbine with our calculation results. It is believed that for the model, the information we need can be accurately obtained from the calculation results.

In this paper, seven working conditions of the synchronous guide vane opening of two runners at rated heads are calculated, respectively, by Fluent ${ }^{\circledR} 2020$ R2: $\mathrm{a}_{1}=142 \mathrm{~mm}$, $\mathrm{a}_{2}=134 \mathrm{~mm}, \mathrm{a}_{3}=126 \mathrm{~mm}, \mathrm{a}_{4}=118 \mathrm{~mm}, \mathrm{a}_{5}=110 \mathrm{~mm}, \mathrm{a}_{6}=102 \mathrm{~mm}, \mathrm{a}_{7}=94 \mathrm{~mm}$. At this stage, the rule of the macro-characteristics with different guide vane openings is studied. Furthermore, unsteady calculation is performed for three operating regimes, namely, the PLP (Part Load Point at $\mathrm{a}_{8}=77 \mathrm{~mm}$ ), the BEP (Best Efficiency Point at $\mathrm{a}_{5}=110 \mathrm{~mm}$ ), and the OLP (Over Load Point at $\mathrm{a}_{1}=142 \mathrm{~mm}$ ) to analyze the relationship between the hydraulic stability and the details of the flow field. Figure 4 shows the placement of eight different 
guide vane openings, where the unsteady calculation starts after the iterative convergence of the steady calculation by using the frozen rotor interfaces and the high-resolution mesh scheme. The calculation time-step is set to 0.00067 , and the total calculation step is set to 7200 steps. Each time-step turns the runner by one degree, which can ensure the accuracy of time discrete calculation. A total of 20 rotation cycles are taken, and the last 5 periods are taken for analysis.

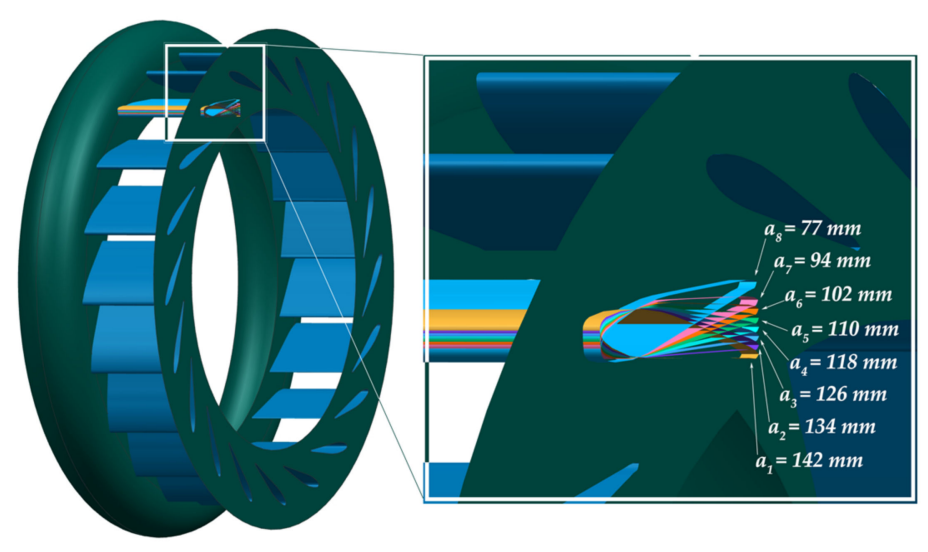

Figure 4. Schematic drawing of different guide vane openings.

\section{Analysis and Discussion of Numerical Results}

3.1. Hydraulic Performance Analysis of Horizontal Axis Double Runner Francis Turbine 3.1.1. Hydraulic Efficiency

Figure 5 shows the variation of hydraulic efficiency of two runners with guide vane opening changes.

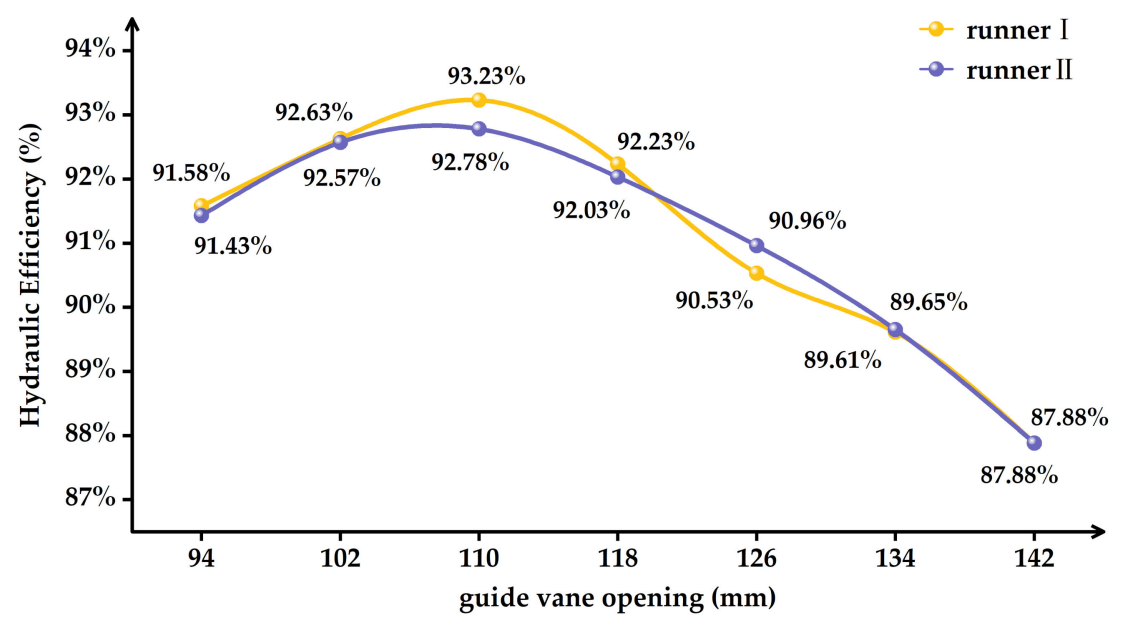

Figure 5. Relationship curve between hydraulic efficiency and guide vane opening.

Obviously, the runner efficiency increases first and then decreases with the decrease of the guide vane opening, and reaches the highest at $\mathrm{a}_{5}=110 \mathrm{~mm}$. Further analysis shows that when the guide vane opening is large, the efficiency of runner I is close to that of runner II, and when the guide vane opening is small, that is, the flow rate of the runner is reduced, the efficiency difference between runner I and II reaches $\pm 0.5 \%$, which is the biggest when the guide vane opening is at $\mathrm{a}_{3}=126 \mathrm{~mm}$ and $\mathrm{a}_{5}=110 \mathrm{~mm}$.

\subsubsection{Flow Rate}

Figure 6 shows the variation of the flow rate of two runners with guide vane opening changes. 


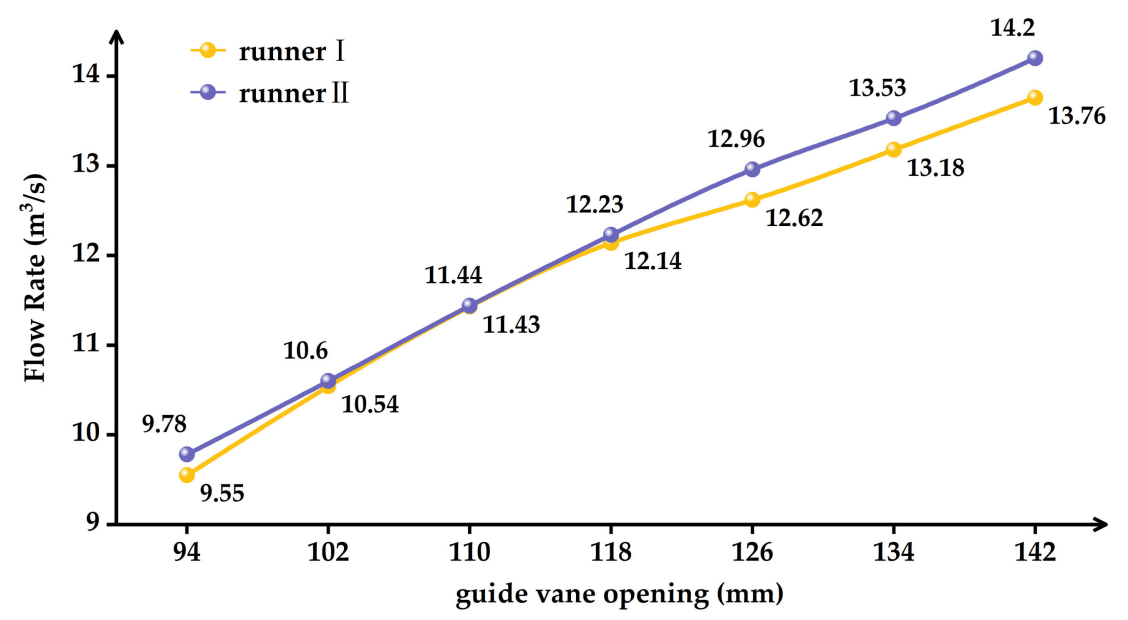

Figure 6. Relationship curve between flow rate and guide vane opening.

Define the flow rate of runners I and II as $\mathrm{Q}_{1}$ and $\mathrm{Q}_{2}$, respectively, and Calculated $\left(Q_{1}+Q_{2}\right) / 2$ as the average flow rate of the runner, half of the flow rate difference between two runners used to define the difference between runner flow and average flow. Furthermore, the efficiency curve is expressed as the average efficiency and its difference, and the relationship between the efficiency and the flow rate with their difference is drawn on a graph (the difference is expressed by the error bar on the graph) shown in Figure 7. As can be seen from the graph of the average efficiency curve, the efficiency difference between the two runners is large with no pattern when the guide vane opening at the operating point is between $110 \mathrm{~mm}$ and $126 \mathrm{~mm}$. However, on the average flow curve, it shows an important feature, which will be discussed in the following.

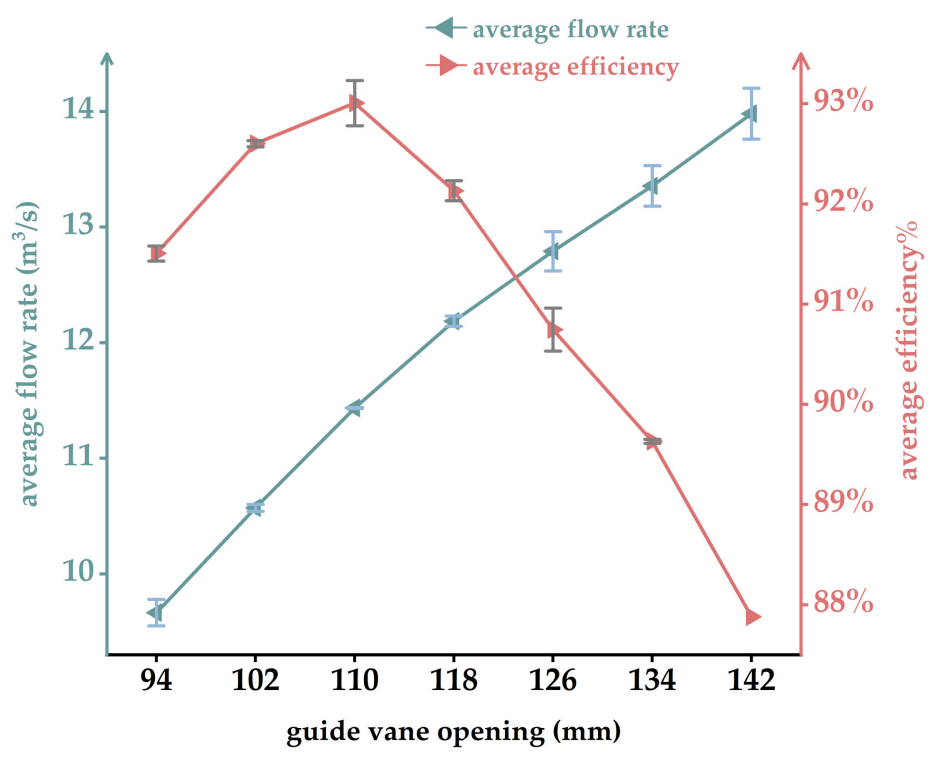

Figure 7. Average efficiency and average flow curve.

Figure 8 shows the relationship between the flow rate difference and runner average efficiency under different guide vane openings. 


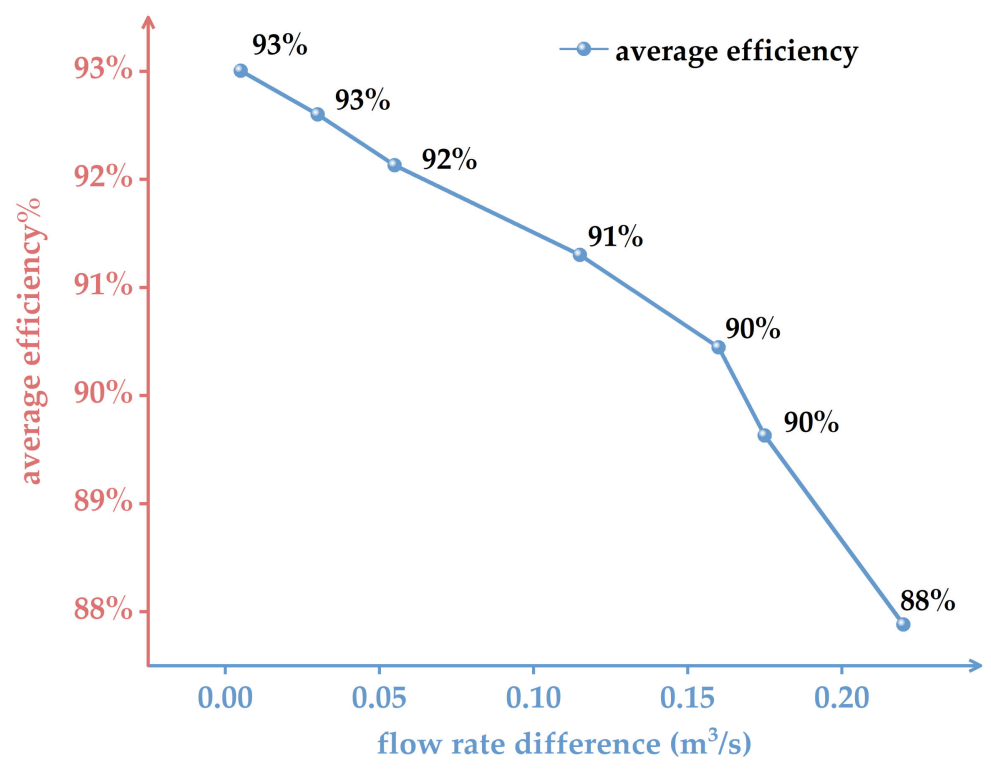

Figure 8. Relationship curve between average efficiency and flow difference.

The graph shows that the average efficiency of the runner I and II is negatively correlated with the flow rate difference between them, that is, the greater the flow difference, the lower the runner efficiency. It is inferred that reducing the flow rate difference between the two runners can improve the overall efficiency of the horizontal axis double-runner Francis turbine.

\subsubsection{Power Output}

Figure 9 shows the variation of the power output of the two runners with the guide vane opening changes.

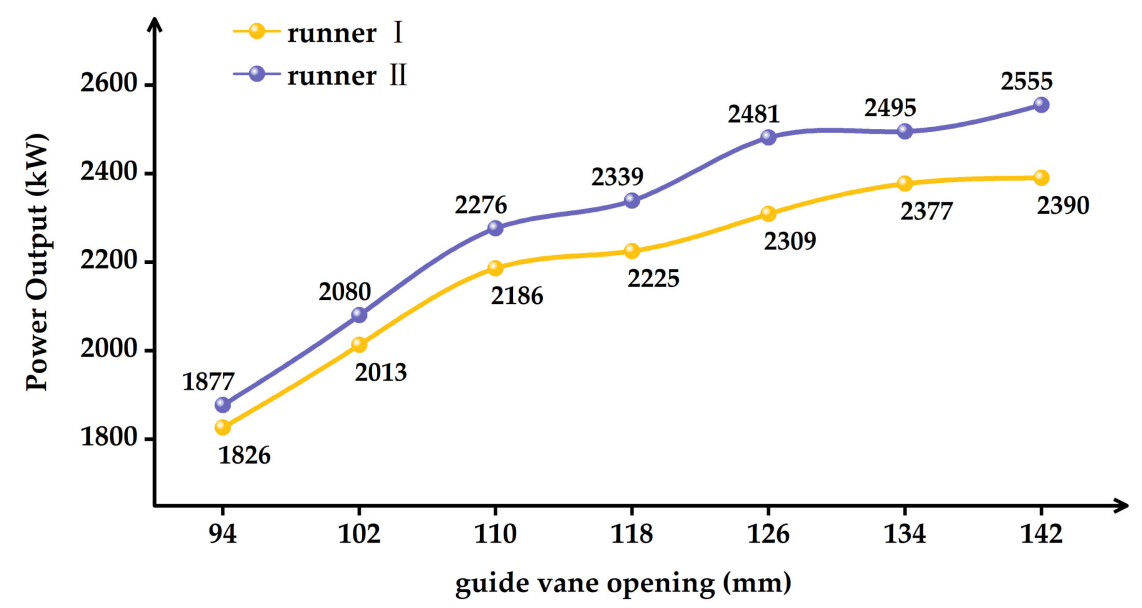

Figure 9. Relationship curve between output and guide vane opening.

The power output of the turbine decreases with the decrease of the guide vane opening. It is worth noting that when the runner efficiency reaches the peak at $\mathrm{a}_{5}=110 \mathrm{~mm}$, and with the guide vane opening reduced, the slope of the curve increases, that is, the power output decreases more sharply. This is because the flow regime in the runner is more turbulent and the flow rate distribution between the two runners interferes with each other. 


\subsection{Analysis of Flow Field of Horizontal Axis Double-Runner Francis Turbine}

\subsubsection{Analysis of The Flow Field in The Runner Areas}

In consideration of the horizontal arrangement of the unit, this section selects five blades at different runner heights for analysis.

Figure $10 \mathrm{~A}(\mathrm{a}, \mathrm{b})$ shows asymmetrical pressure distributions on the surface of the runner's blades arranged along a horizontal axis. A typical "vertical-direction" distribution is first illustrated in Figure 10 under OLP conditions. Along the direction of gravity, the pressure distribution on both sides of the runner blades shifted along the direction of flow. During the migration process, the low-pressure range on the blade surface becomes larger, and the negative pressure area is also evident on the pressure side of the blade below the main shaft (hereinafter referred to as "the bottom"). By observing the local magnified figure, it is found that the pressure isolines at the inlet edge of the blades above the main shaft (hereinafter referred to as "the top") are dense, which indicates that there is a large pressure gradient, and the value of high-pressure force and pressure gradients at this point change with the height of the blades. The extremum pressure on the blade surface decreases with lower height, which is contrary to the pressure distribution under gravity. The negative pressure distribution at the outlet side of the suction side of the blade is also not in accordance with the pressure distribution under gravity.

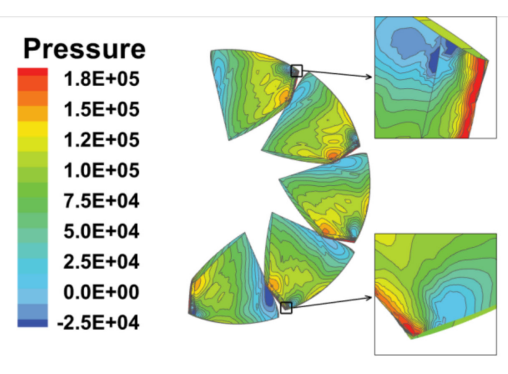

(a)

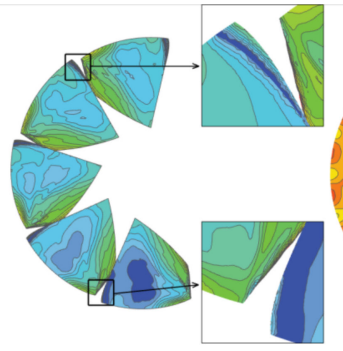

(b)

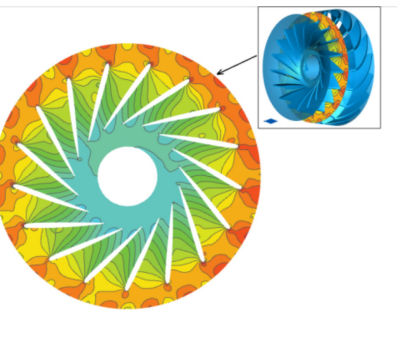

(c)

(A) Pressure contour of runner I.

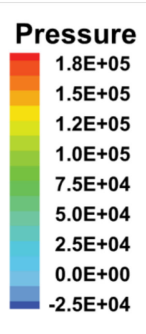

(a)

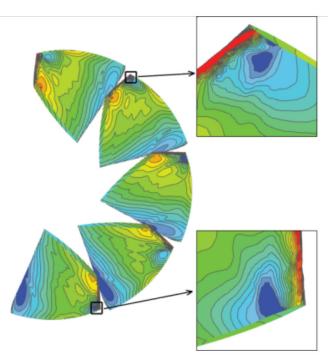

.

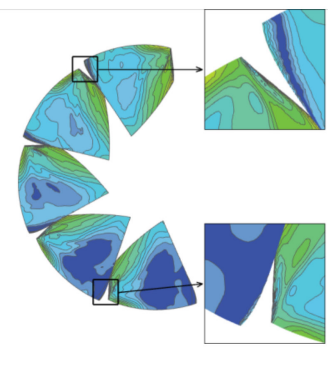

(b)

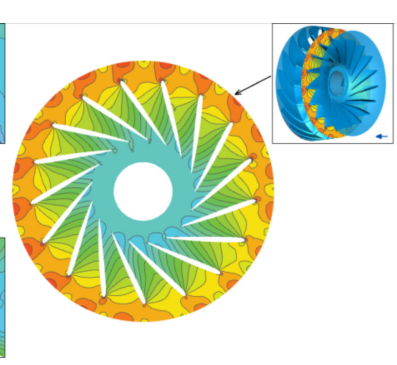

(c)

(B) Pressure contour of runner II.

Figure 10. Pressure contour of the runner blade and runner flow channel under OLP $\left(\mathrm{a}_{1}=142 \mathrm{~mm}\right)$. (a) Pressure side of blade; (b) suction side of blade; (c) runner flow channel.

Figure $10 \mathrm{~A}(\mathrm{c})$ shows that, unlike the pressure distribution on the blade surface, the pressure distribution in the runner channel is relatively symmetrical and the pressure gradient is uniform. There is a small area of abnormal pressure near the runner top area and runner blade inlet edge, which shows that the flow regime is poor. According to the pressure distribution of the channel section, the flow into the runner impinges on the suction side of the vane, and partial reflow occurs, causing an abnormal vortex in the channel. In addition, the pressure distribution on the outer side of the runner outlet (i.e., the central area of the cross-section) again shows a trend that is contrary to the influence of gravity, which, we believe, is related to the arrangement of the draft tube of the turbine. This conclusion will be supported by Section 3.2.2 of this chapter. 
Comparing the pressure distribution of the two runners in Figure 10A,B, the pressure at the inlet and outlet of runner II is lower, and the area of low pressure on the blade surface is bigger, especially on the suction side of the blade, where the area of negative pressure reaches $2 / 3$ of the blade area. In addition, from the pressure gradient distribution of the runner section, we can see that the flow regime at the top of runner II is good and there is no abnormal pressure gradient, similar to the top area of runner I.

Combined with the analysis of the hydraulic performance data of the hydraulic turbine in Section 3.1, under the condition of the OLP $\left(a_{1}=142 \mathrm{~mm}\right)$, the flow rate and the power of runner I are lower than that of runner II while the efficiency of the two runners is close, and because the efficiency is affected by the flow rate, the water head, and runner power, the effective water head, $H_{a}$, used by the two runners is different. The difference between the effective head and the effective flow rate of the two runners is reflected in the flow regime and pressure distribution of the runner in this section. At this point, affected by the local adverse flow regime, the backflow in runner I causes the reduction of its effective flow rate. In addition, compared with runner II, the inlet and outlet of runner I have higher pressure extremes, but the pressure gradient is smaller than that of runner II, and the difference in inlet and outlet pressure of the runner can be expressed as the effective head $H_{a}$, so the effective head $H$ of runner I is lower than that of runner II, and the efficiency of runner II is higher than that of runner I.

As shown in Figure 11A,B, according to the average efficiency curve of Figure 7 in Section 3.1, the average efficiency of the runner is the highest under the BEP $\left(a_{1}=110 \mathrm{~mm}\right)$. At this time, the pressure distribution on the blade surface is basically the same as that in the case of the OLP. Different from the large flow condition, the pressure contour lines on the blade surface are straighter, the density of contour lines distribution is more uniform and the pressure gradient jumps less. When looking at the flow channel of the two runners (c), the pressure contour lines are arranged in order, the pressure distribution is relatively uniform, and there is no abnormal pressure area (the flow regime turbulence at the top of runner I under the condition of the OLP) inside the runner channel, which indicates that the flow regime in the runner channel is good under this condition.

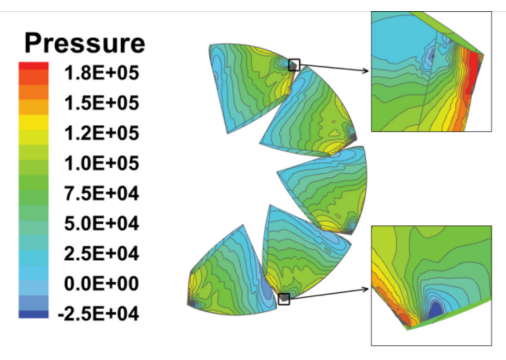

(a)

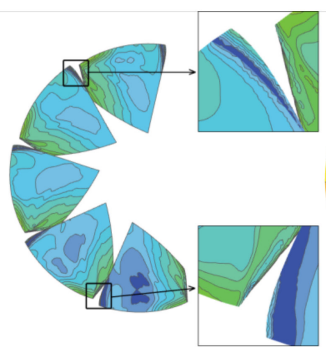

(b)

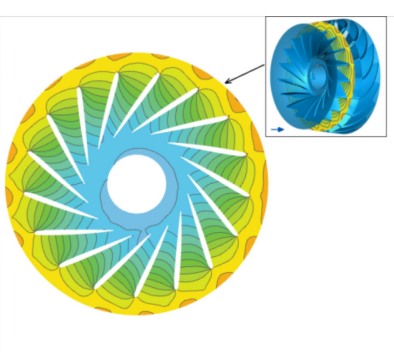

(c)

(A) Pressure contour of runner I

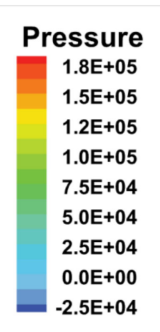

(a)

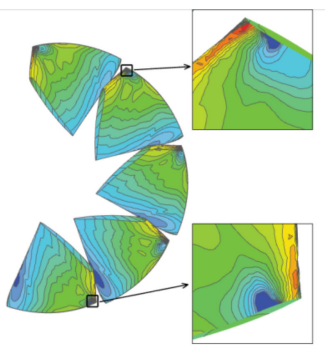

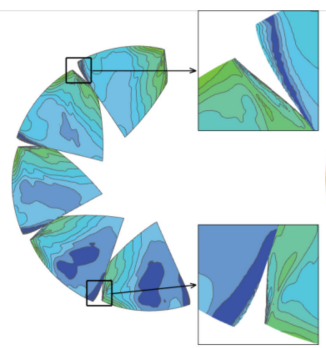

(b)

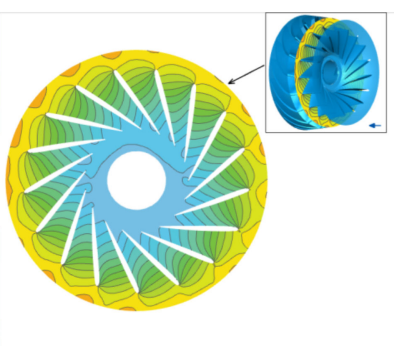

(c)

(B) Pressure contour of runner II

Figure 11. Pressure contour of runner blade and runner flow channel under BEP $\left(a_{5}=110 \mathrm{~mm}\right)$. (a) Pressure side of blade; (b) suction side of blade; (c) runner flow channel. 
Comparing Figure 11 with Figure 10, Unlike the OLP, under the condition of the BEP, the pressure distribution between the two runners tends to be the same, and the area of low pressure on the pressure side and the area of negative pressure on the suction side are respectively the same. This phenomenon is related to the previous inference that "if the two runner flows tend to be the same, the efficiency will be improved". When the flow rate tends to be the same, the flow difference between the two runners decreases, which causes the pressure distribution tend to be the same, too. When equilibrium is maintained, the flow regime in the runner is improved, the unbalance of the two runners with respect to the main shaft is almost eliminated, and the efficiency of the two runners is increased. Although the pressure distributions in the two runners and on the surface of the blades tend to be the same under the BEP, there is still a difference in the pressure between the two runners at the exit cavity of the runner. By calculating the surface average pressure of the interface of the runner outlet, the resulting value of the runner II is about $15 \mathrm{KPa}$ lower than that of runner I, and the efficiency difference between the two runners also reaches the maximum, indicating that the flow inside the runner is really affected by the flow of water outside the runner's outlet.

As shown in Figure 12A,B, when the guide vane opening is adjusted to the condition of PLP $\left(a_{8}=77 \mathrm{~mm}\right)$, the efficiency and flow rate of the two runners are $86.6 \%$ and $8.17 \mathrm{~m}^{3} / \mathrm{s}$ of runner I, and $86.7 \%$ and $7.77 \mathrm{~m}^{3} / \mathrm{s}$ of runner II, respectively. The efficiency of the two runners is close, but the flow rate is different. The observation in Figure $12 \mathrm{~A}(\mathrm{a}, \mathrm{b}), \mathrm{B}(\mathrm{a}, \mathrm{b})$, and blade pressure distribution compared with the previous two conditions changed greatly. Firstly, the pressure contour lines of the pressure side of the blade is no longer parallel to the inlet and outlet edge of the blade, but a circular negative pressure gradient with concentric circle distribution appears near the outlet edge of the blade and the runner crown, indicating that the flow in the runner is significantly affected by fluid flow outside the runner outlet. Secondly, compared with the large flow condition, the negative pressure area on the suction side of the blade is reduced, the pressure contour is locally dense and messy, and the pressure gradient distribution is more uneven. It can be seen that the flow regime in the runner is poor and the pressure difference between the inlet and outlet of the runner is further reduced. In Figure 12A(c),B(c), as the fluid goes deep into the runner, the pressure contour in the runner channel no longer pushes forward to the outlet regularly, and the obvious point-like low-pressure area can be seen in the middle of the inlet of the channel. According to Guo's [38] research, when the point deviates from the optimal working condition area, there will be an obvious blade vortex in the runner channel. The flow point-like low-pressure area shown as the graph of the cross-section is consistent with the flow channel pressure contour when the channel vortex occurs in articles [38]. It can be judged that when the guide vane opening is at $\mathrm{a}_{8}=77 \mathrm{~mm}$, the runner generates and develops a more significant channel vortex. In addition, a low-pressure zone which accounts for about $1 / 4$ of the circle is also derived from the outlet cavity of runner II.

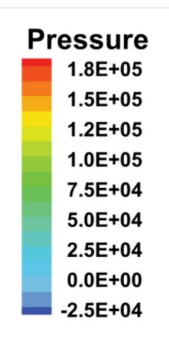

(a)

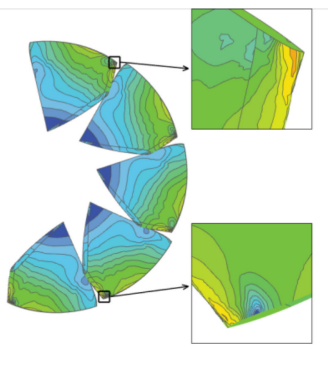

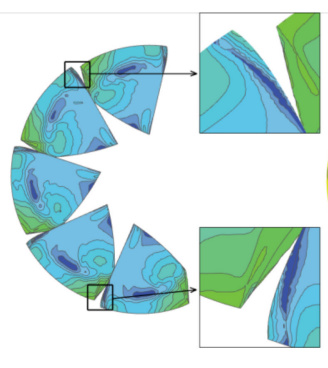

(b)

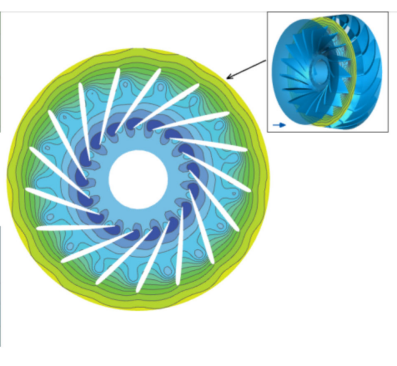

(c)

(A) Pressure contour of runner I.

Figure 12. Cont. 


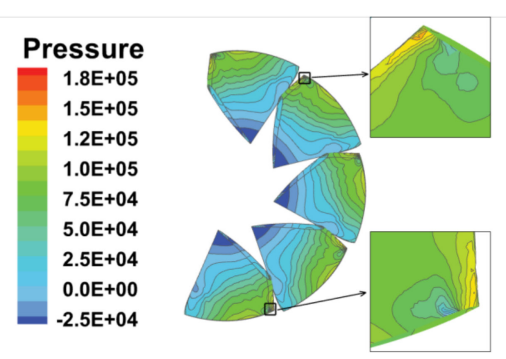

(a)

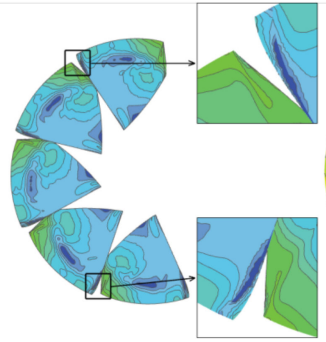

(b)

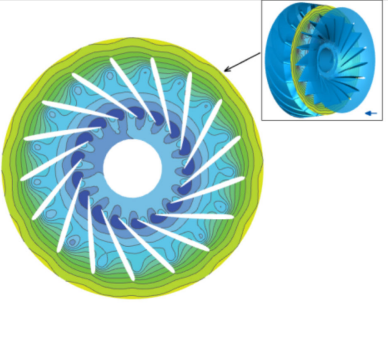

(c)

(B) Pressure contour of runner II.

Figure 12. Pressure contour of runner blade and runner flow channel under PLP $\left(a_{8}=77 \mathrm{~mm}\right)$. (a) Pressure side of blade; (b) suction side of blade; (c) runner flow channel.

\subsubsection{Analysis of Flow Field in Draft Tube}

(1) The collection elbow of draft tube

Based on the above analysis of the pressure contour inside the runner, the uneven pressure distribution in the runner is not only due to gravity, but also from the downstream flow regime. In this paper, the outlet of the two runners is arranged in an opposite direction. The two runners share the draft tube through a collection elbow. In order to explore how the flow at the outlet of the runner affects the flow in the runner, the flow in the collection elbow under different working conditions is analyzed in detail.

Figure 13 shows the sectional streamline, the surface velocity vector, and pressure contour of the collection elbow under three working conditions, respectively. As can be seen in Figure $13 \mathrm{~A}(\mathrm{a})$, the main stream of water flowing out of the two runners converges roughly at the junction of the left and right elbows, and forms a local high-pressure zone when it collides with the wall between the two elbows, with a relatively large low-speed backflow (i.e., the dead water zone) at the inner side of the elbows near the draft tube cone. The velocity vector distribution in Figure $13 \mathrm{~A}(\mathrm{~b}, \mathrm{c})$ fully illustrates the vortex direction and development trend of the backflow, which occurs in a low-pressure region with a wide range of distribution, and corresponds to the initial development region of the dead water zone. Through the analysis of the flow at the outlet of the runner, it is found that the rotation direction of the backflow is almost the same as that of the corresponding runner, because the water flow from the lower part of the draft tube converges with the flow of the runner outlet at a certain circumferential velocity near the inner side of the collection tube, changes the flow direction, flows toward the lower end of the draft tube again, and forms a "dead water zone".

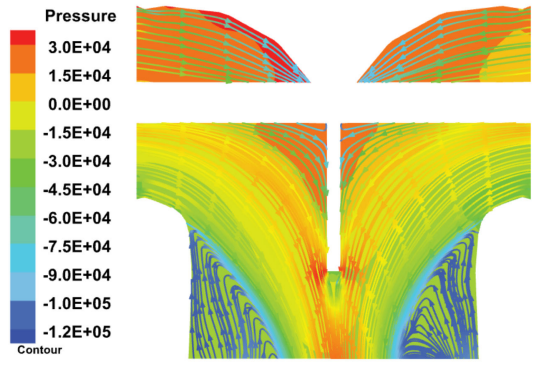

(a)
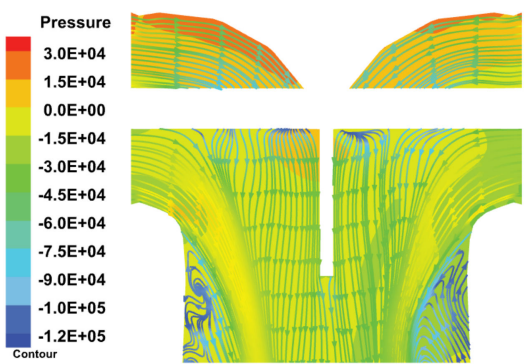

(a)
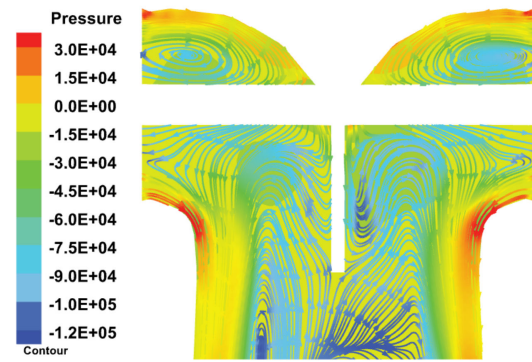

(a)

Figure 13. Cont. 


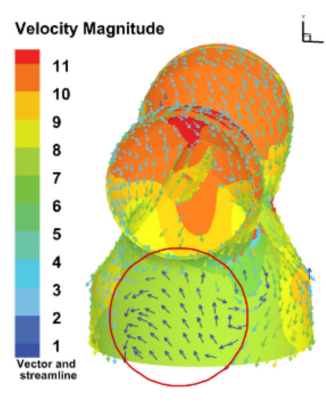

(b)

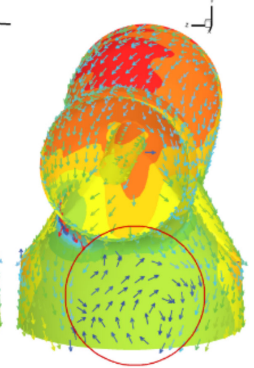

(c)

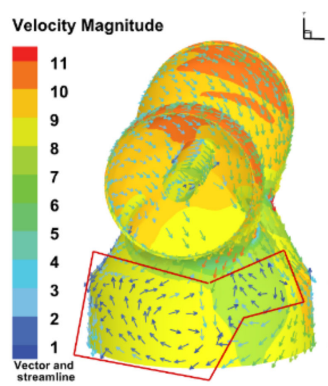

(b)

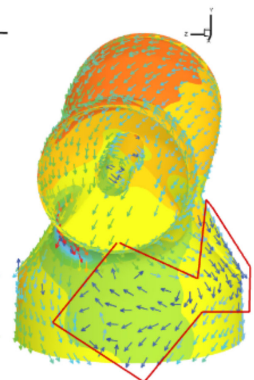

(c)

(B) BEP condition $\left(\mathrm{a}_{5}=110 \mathrm{~mm}\right)$

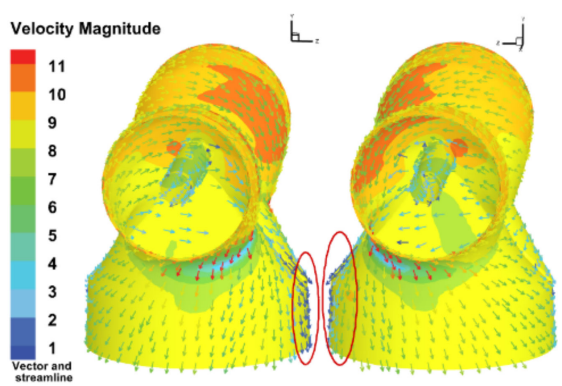

(b)

(c)

(C) PLP condition $(\mathrm{a} 8=77 \mathrm{~mm})$

(A) OLP condition $\left(\mathrm{a}_{1}=142 \mathrm{~mm}\right)$

Figure 13. Streamline diagram and surface velocity vector diagram of collection elbow under different working conditions. (a) Streamline of cross-section of collection elbow; (b) velocity vector diagram from runner I side view; (c) velocity vector diagram from runner II side view.

Comparing the mainstream, backflow, and pressure distribution in the collection elbow shown in Figure 13, it is found that under the condition of the OLP, the mainstream from the outlet of both sides of the elbow converges into an earlier bundle, and the backflow mainly occurs in the lower part of the inner side of the elbow, the pressure distribution is roughly bounded by the mainstream, the inner side is a positive pressure area, and the outer side is a negative pressure area. Under the BEP, the mainstream of both sides of the runner outlet is located in the central area of their respective flow channels, and the flow of the backflow of the draft tube on both sides is suppressed by the two main streams, so that the area of the backflow of the inner side of the elbow is reduced, the area of a negative pressure zone on the outer side is narrowed, and the pressure gradient distribution in the bend is relatively balanced. Under the condition of the PLP, because the flow of the turbine is approaching the minimum flow rate, the backflow flows the whole elbow, while the flow of the outlet of the runner is flowing close to the wall, and the area of high-pressure impact appears on the inner side of the collection elbow. Compared with the figure drawn of the velocity vector, with the decrease of the guide vane opening, the low-speed backflow region develops from the inner side of the elbow to the joint of the two sides, which is the result of the movement of the mainstream direction of the runner outlet flow in the upper elbow of the draft tube, and also shows that the change of the mainstream direction can significantly affect the size of the dead water zone and the development of the backflow in the draft tube. For large flow rates, a large area of low-pressure backflow is attached to the inner side of the elbow, which is closer to the bottom of the runner, thus greatly lowering the pressure in the bottom area of the runner. With the decrease of the guide vane opening, the mainstream of the runner outlet moves from the center to the periphery, the range of low-pressure area becomes smaller and gradually transfers to the center of the flow channel, and finally becomes a negative-pressure area with circumferential distribution in the middle part of the runner at a low flow condition.

Based on the analysis of the flow field in the collection elbow area, it is found that the flow direction and convergence form of the mainstream in the elbow area dominate the distribution of the backflow, and affect the distribution of the outlet pressure of the runner at the same time. The distribution of the backflow is the key factor affecting the dead water zone, while the pressure distribution of the outlet of the runner affected by the flow regime of the collection elbow is further fed back to the upstream of the runner, which aggravates the unbalance of the flow in the horizontal-axis runner. Therefore, the abnormal phenomenon in Section 3.2.1 that the variation of the pressure of the runner blade surface between different blades is opposite to the gravity effect appears. Because the two runners share the same draft tube, the flow in the draft tube is more complicated, and the flow regime in the draft tube affects the upstream flow regime of the runner. It is necessary to 
further explore the flow regime of the rear of the draft tube and the propagation of pressure pulsation caused by the vortex inside the turbine.

(2) Elbow and diffusion section of draft tube

Based on the analysis of the flow in the collection elbow, the flow in the elbow section and the diffusion section of the draft tube will be affected by the flow in the upstream runner by the convergence form of the main current at the exit of the runner.

The flow regime of the draft tube flow field under different working conditions is analyzed. The section plane shown in Figure 14 is the inlet of the lower section of the draft tube and the outlet of the collection elbow. According to the velocity vector distribution in Figure $14 \mathrm{~A}$, under the condition of the OLP, the velocity of the cross-section has a reversed flow state near both sides of the collection elbow, and the pressure in the corresponding region has negative pressure below $-30 \mathrm{KPa}$. As the flow rate changes, in Figure 14B, the backflow region is compressed, and the backflow on one side is separated by the mainstream impact, and the pressure value rises and tends to equilibrium. When the flow rate is further reduced to the PLP, as shown in Figure 14C, a large area of backflow occurs in the center of the channel, and the low-pressure region is squeezed to the side of the channel by the mainstream.

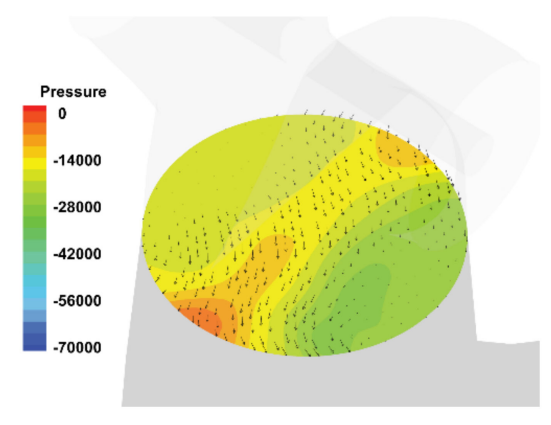

(A) OLP condition $\left(\mathrm{a}_{1}=142 \mathrm{~mm}\right)$

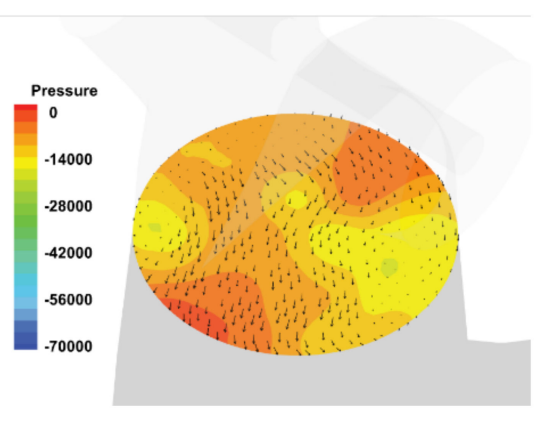

(B) BEP condition $\left(\mathrm{a}_{5}=110 \mathrm{~mm}\right)$

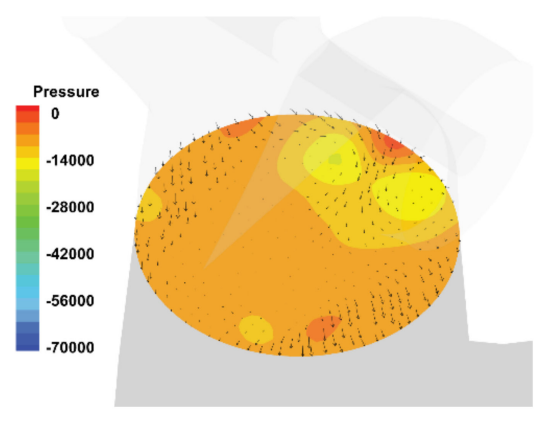

(C) PLP condition $(\mathrm{a} 8=77 \mathrm{~mm})$

Figure 14. Velocity vector diagram and pressure contour of the inlet section of the draft tube under different working conditions.

Combined with the streamline diagram in Figure 15, it is found that the main stream of the draft tube under all working conditions basically continues the development trend of the mainstream at the outlet of the collection elbow. Under the condition of the OLP, the mainstream is in the center of the channel, close to the inside of the elbow section, and enters the diffusion section, and a small part of the flow develops into a small vortex. At the BEP, there are two parallel mainstreams that do not interfere with each other. The bottom mainstream flows out through the elbow section, and the top mainstream develops into a primary vortex after entering the diffusion section. Under the condition of the PLP, the mainstream is close to the wall of the draft tube, and part of it is separated from the wall after entering the diffusion section, and becomes the primary vortex. There are a large number of vortices and backflow at the elbow under the condition of the OLP and the PLP, which fills almost $90 \%$ of the area of the whole draft tube channel especially at the PLP, while the elbow under the condition of the design only has a small Eddy with incomplete development. Focusing on the side of the diffusion section, the flow regime of the OLP is better than the other two conditions, and with the decrease of the flow rate, the primary vortex begins to develop at the top of the diffusion section under the condition of the BEP. When the flow rate decreases to the PLP, the primary vortex of the elbow section is connected with the primary vortex of the diffusion section, and a large number of secondary vortices is derived, which makes the upper part of the diffusion section almost completely filled with vortices and reflux. 


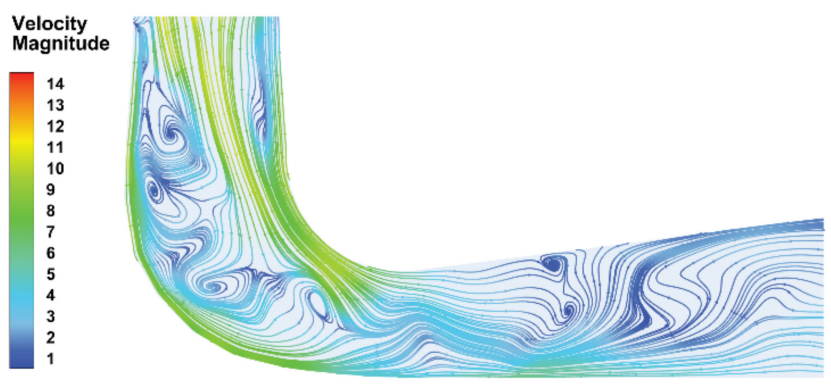

(A) OLP condition $\left(\mathrm{a}_{1}=142 \mathrm{~mm}\right)$

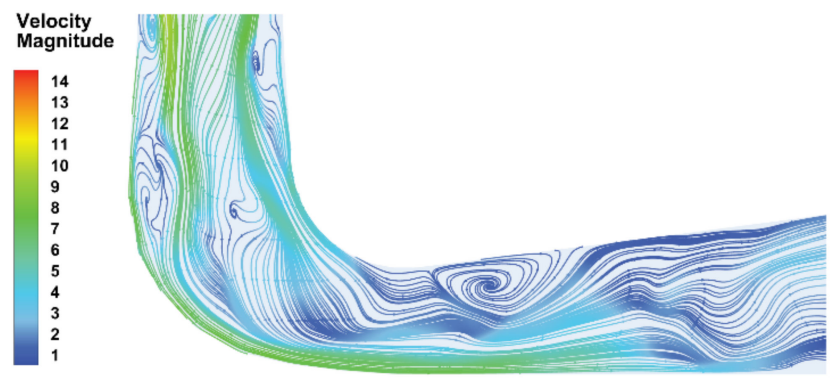

(B) BEP condition $\left(\mathrm{a}_{5}=110 \mathrm{~mm}\right)$

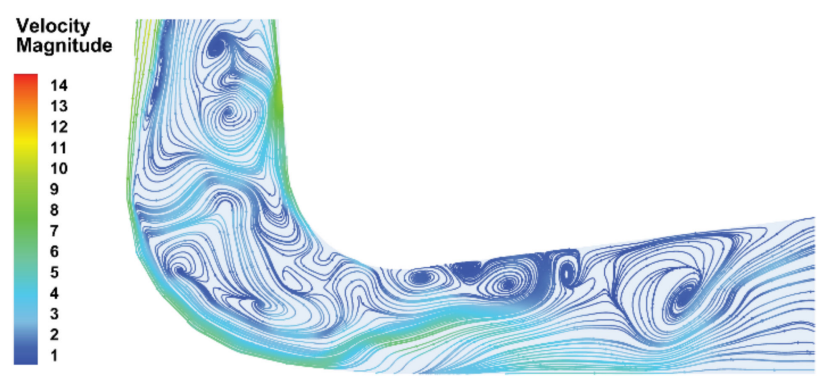

(C) PLP condition $\left(\mathrm{a}_{8}=77 \mathrm{~mm}\right)$

Figure 15. Streamline of central section of draft tube under different working conditions.

A large number of vortices make the energy in the draft tube unable to be effectively recovered, and the abnormal pressure pulsation generated by the vortex core and vortex rope will adversely affect the whole flow passage of the turbine. Therefore, optimizing the mainstream flow in the draft tube and improving the distribution area of the backflow will become the key direction to increase the efficiency of the double-runner and optimize its internal flow regime.

\subsection{Pressure Pulsation Analysis of Horizontal Axis Double-Runner Francis Turbine}

The above analysis shows us the steady-state information of the flow field inside the turbine, which is usually not enough to reflect the significant characteristics of the turbine operation. In the unsteady parameters of the hydraulic turbine, the pressure pulsation characteristic is an important item to measure the working state of the hydraulic turbine. In the normal operation of rotating machinery, instantaneous fluctuation of pressure is more or less accompanied, and the main pulsation source comes from the rotation of runners and the eccentric movement of vortex ropes in the draft tube. In almost all cases, the pressure pulsation information associated with the rotation of the runner usually does not adversely affect the operation of the turbine, and the vortex line moving with limited eccentricity is not harmful. However, the turbine often needs to change between different flow rates to cope with external flow conditions or load changes. Previous studies have shown that when the turbine deviates from the design working condition, there will be 
unusual pressure pulsation, which either comes from the rotor-stator interference outside the runner or derives from the large low-frequency vortex rope movement of the draft tube. They can cause intense mechanical vibration, deafening noise, or even worse, risk of shaft breakage. All these seriously threaten the production safety of hydropower stations. The analysis of amplitude-frequency characteristics of measuring points in different areas can help us collect the information of high- and low-frequency signals in turbine runners better, so as to weaken the influence of abnormal pressure pulsations in an accurate position. This paper studied the amplitude-frequency characteristics of the pressure pulsation of the double-runner turbine, and the analysis results may show us some valuable discoveries and reveal the characteristics of the pressure pulsation of double-runner turbines, which can provide an effective reference for future research. Figure 16 shows the monitoring points inside the turbine.

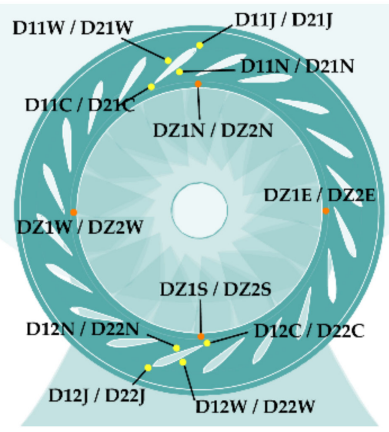

(a)

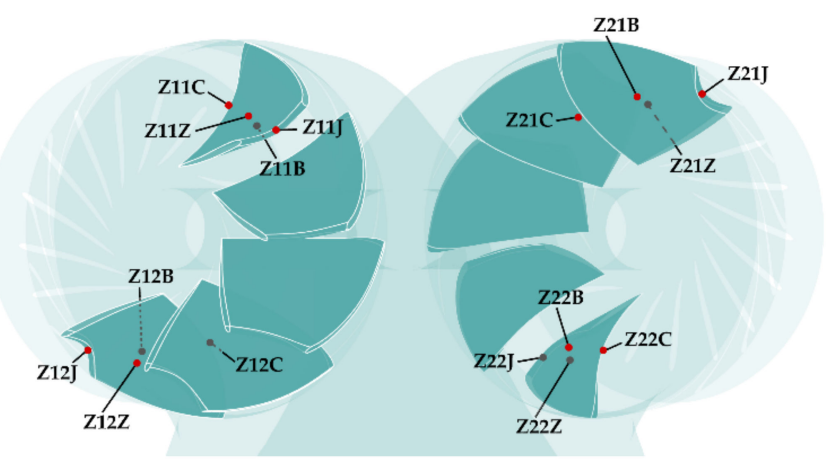

(b)

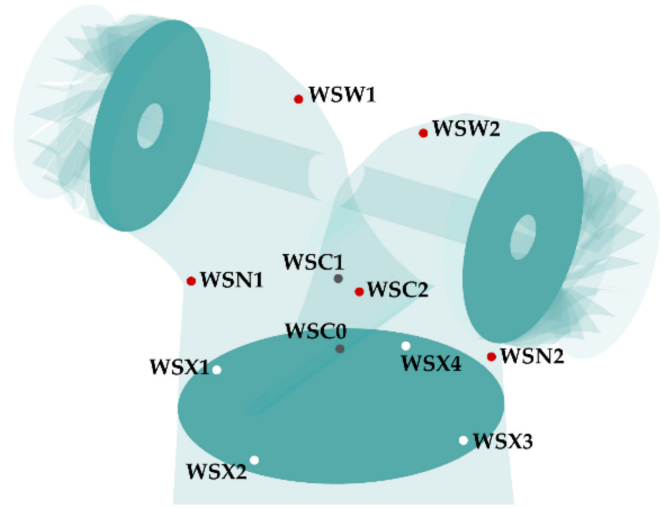

(c)

Figure 16. Pressure pulsation monitoring points in each part. (a) Monitoring points in guide vanes and bladeless zones; (b) monitoring points in runner blades; (c) monitoring points in the collection elbow and its outlet. 


\subsubsection{Analysis of Pressure Pulsation in Guide Vane}

As shown in Figure 16a, two guide vanes with different installation heights are selected on the runner I side and runner II side of the ring, and a monitoring point is set on the inlet, outlet, and inner and outer sides of each of the guide vanes, respectively; four monitoring points with different heights are set along the circumference of the bladeless zone between the runner inlet and the guide vanes outlet.

As can be seen from Figure 17, the pressure pulsation frequency on the guide vane surface is mainly low-frequency, and the runner blade passing frequency is $17 f n$ (hereinafter referred to as the RBPF) and its frequency doubles. On the whole, the low-frequency signal is the main part of the pressure pulsation signal on the surface of the guide vane. The BPF signal gradually weakens with the decrease of the flow rate, and the obvious double frequency appears under the condition of the OLP. As for the RBPF signals, among the different monitoring points on the guide vane surface, the backside of the guide vane and the outlet of the guide vane are closer to the runner, so the blade frequency signal is stronger than other monitoring points. According to the information in the figure, the amplitude relation of the four monitoring points on the guide vane surface is as follows: backside > outlet edge $>$ frontside $>$ inlet edge, and this relationship is not related to the guide vane opening and the guide vane placement position. We think that this distribution is related to the opposite arrangement of the double runner. The amplitude and distribution of lowfrequency signals will change when the working conditions change. The low-frequency signal will be analyzed in different working conditions.

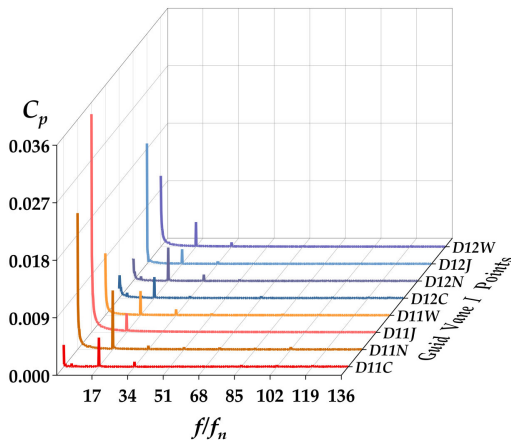

(a)

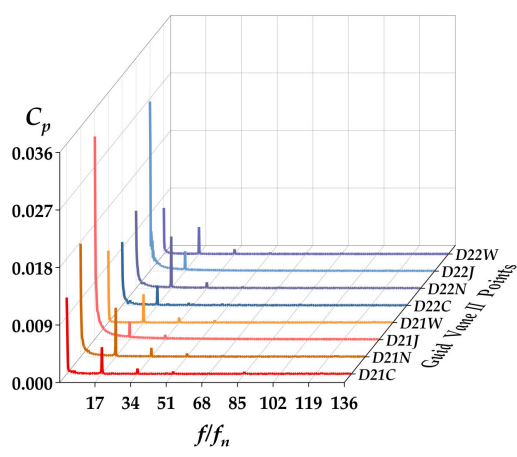

(b)

(A) OLP condition ( $\mathrm{a}_{1}=142 \mathrm{~mm}$ )

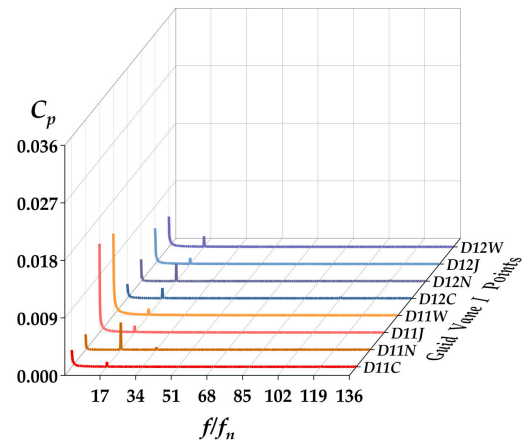

(a)

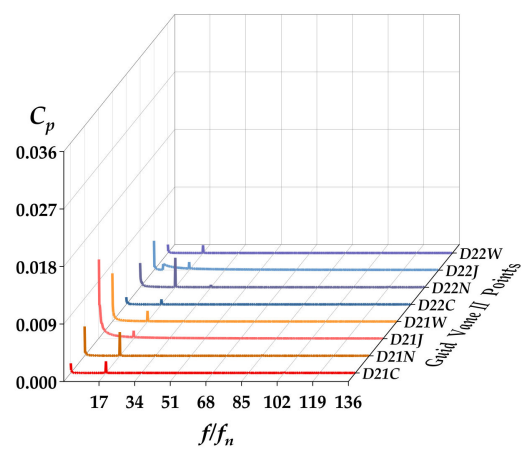

(b)

(B) BEP condition $\left(\mathrm{a}_{5}=110 \mathrm{~mm}\right)$

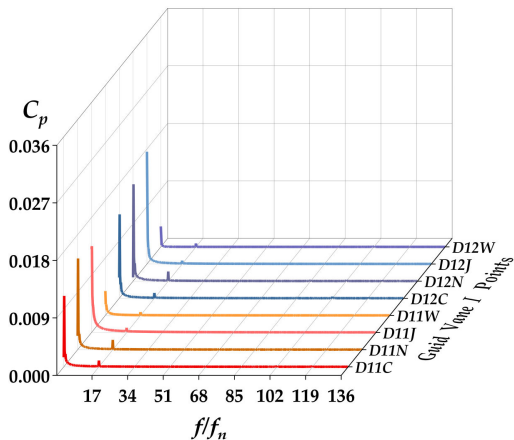

(a)

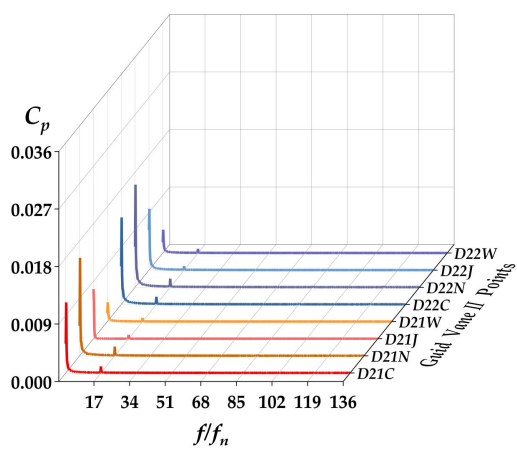

(b)

(C) PLP condition $(\mathrm{a} 8=77 \mathrm{~mm})$

Figure 17. Spectrum of pressure pulsation at guide vane monitoring points under different working conditions. (a) Data from guide vane of the runner I side; (b) Data from guide vane of the runner II side.

Under the condition of the OLP, the low-frequency amplitude fluctuates greatly among different monitoring points of the same guide vane. The measuring point with the highest amplitude is located at the inlet of the guide vane, and the low-frequency pressure pulsation 
coefficient $C p_{\max }$ reaches 0.034 . The average amplitude of the runner II side is higher than that of the runner I side, while the average amplitude of the pressure pulsation on the surface of the high-position guide vane on both sides is higher than that of the low-position guide vane.

Under the BEP, the flow regime in the turbine is good, and the amplitude of the low frequency signal is low as a whole. The highest average amplitude of a single guide vane appears at the low-position guide vane of both sides of the runner, and the highest amplitude is still at the inlet point of the guide vane. At this time, the low-frequency pressure pulsation coefficient $C p_{\max }$ is 0.014 .

Under the condition of the PLP, the low-frequency amplitudes of all monitoring points except the inlet of the guide vane are higher than those under the BEP. The average amplitude of the runner I side is higher than that of the runner II side, and the highest amplitude moves to the monitoring point at the backside of the guide vane. The average amplitude of the low-position guide vane is close to and slightly higher than that of the high-position guide vane.

Synthetically, the pressure pulsation signal on the guide vane surface will regularly migrate between different guide vane bodies of different runners with the change of flow rate. Starting from the OLP, as the flow rate decreases, the low-frequency pressure pulsation amplitude decreases first and then increases as a whole, and the strong pressure pulsation signal area of the guide vane surface moves from the runner I side to the runner II side and changes from the low-position guide vane to the high-position one. The same as what we have concluded from the analysis of the flow field in Section 3.2, the flow regime of the runner is affected by the flow in the draft tube, and the influence is transferred to the upstream of the guide vane region, so that the pressure pulsation signal of the guide vane moves to different heights when the flow rate conditions change.

Figure 18 is the spectrums of the pressure pulsation point in the bladeless region. According to the article [22], the high-frequency pulsation of rotor-stator interference originates from the bladeless region and can be transmitted upstream or downstream, which is one of the reasons for the abnormal pressure pulsation of the hydraulic turbine. It can be seen from the graph that the pressure pulsation signal between the guide vane and the runner is mainly low-frequency, and the RBPF is secondary. Under the condition of sufficient flow, there is also a weak, discrete, high-frequency quantity that decreases with the change of flow rate. The low-frequency signal mainly concentrates on the top and bottom of the runner under the condition of a large flow rate in the bladeless area. The amplitude of the pressure pulsation of low frequency and the RBPF attenuates with changes in the flow rate, and reaches a high level in the condition of the PLP. The average amplitude of each monitoring point is about two to four times that at the OLP, and five to eight times that at the BEP. Through the analysis of the typical values of high-frequency components, it is found that the complex high-frequency components are mainly composed of 1.17 times RBPF and its harmonics 2.34 times, 3.51 times, and 4.68 times RBPF. Further analysis shows that 1.17 times RBPF and the corresponding harmonics are the guide vane passing frequency $20 \mathrm{fn}$ and its doubling frequency. The guide vane passing frequency (hereinafter referred to as the GVPF) and the runner blade passing frequency are mixed to form the combined blade-passing frequency.

The results show that the blade-passing frequency and the discrete quantity of highfrequency pressure pulsation in the bladeless region attenuates with the lower flow rate, and the low-frequency pressure pulsation signal is the strongest under the condition of the PLP. In addition, the discrete high-frequency quantity caused by rotor-stator interference occurs in the bladeless region in a complex form of combined blade-passing frequency. 


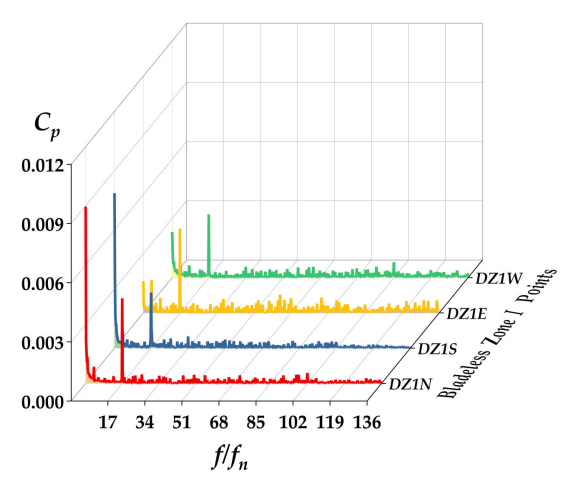

(a)

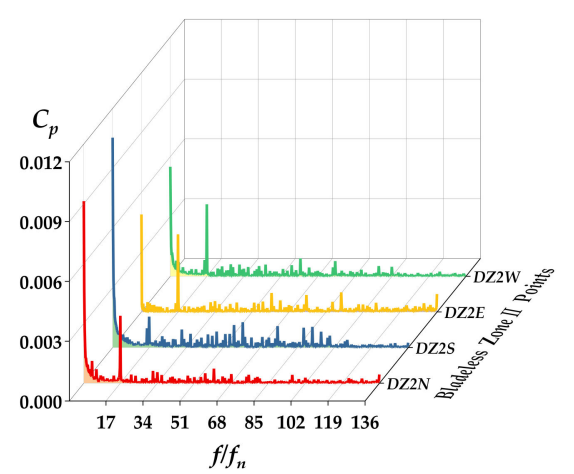

(b)

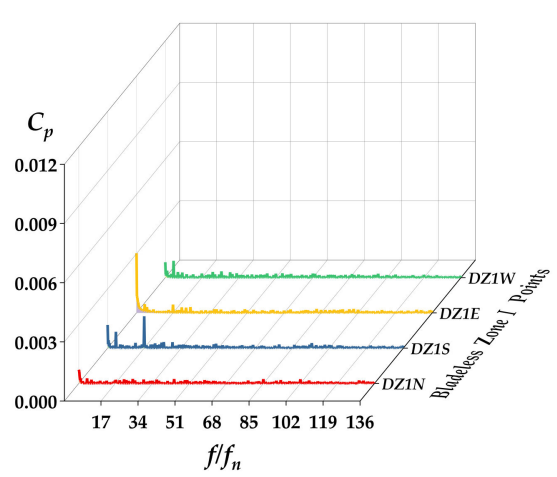

(a)

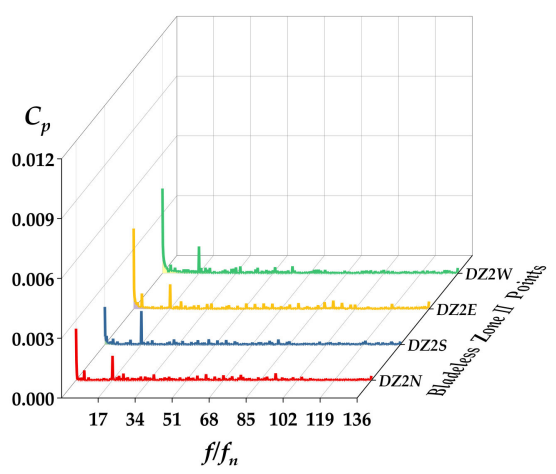

(b)

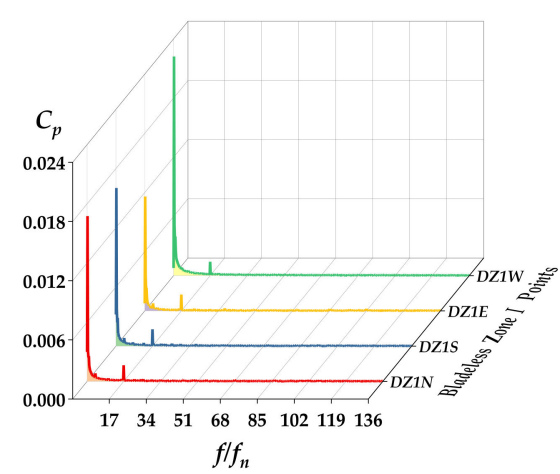

(a)

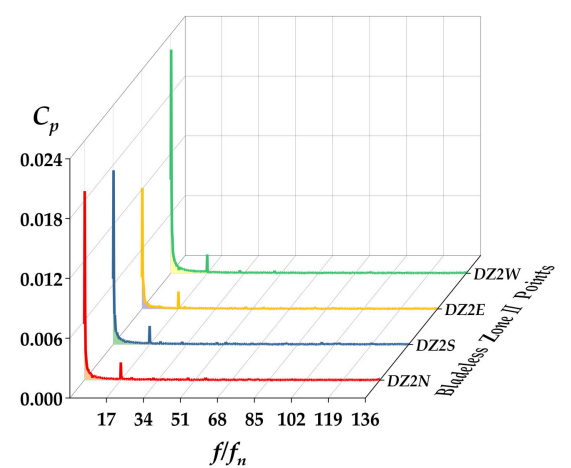

(b)

(C) PLP condition $(\mathrm{a} 8=77 \mathrm{~mm})$

(A) OLP condition ( $\mathrm{a}_{1}=142 \mathrm{~mm}$ )

(B) BEP condition $\left(\mathrm{a}_{5}=110 \mathrm{~mm}\right)$

Figure 18. Spectrum of pressure pulsation at bladeless zone monitoring points under different working conditions. (a) Data from bladeless zone of the runner I side; (b) data from bladeless zone of the runner II side.

\subsubsection{Analysis of Pressure Pulsation in Runner Region}

Figure 19 is the spectrums obtained at the monitoring points of the runner blade surface under different flow rates. On the whole, the basic frequency of pressure pulsation on the surface of the runner blade is the RBPF, and there is a high value of blade-frequency doubling, and the amplitude of the pressure pulsation of the RBPF and its doubling attenuates with the decrease in flow rate. The low-frequency pressure pulsation signal with the same amplitude as the guide vane monitoring point can be detected at the PLP.

Compared with the RBPF signals of two runners, the maximum amplitude of the RBPF pressure pulsation on the runner I side is higher than that on the runner II side, and there is a high-frequency rotor-stator interference pressure pulsation at several monitoring points, which shows that the pressure pulsation originates from the bladeless zone that can spread to the runner inside. At different monitoring points on the same blade surface, the amplitude of the pressure pulsation signal measured at the pressure side and suction side of the blade is much higher than that at the inlet edge and outlet edge, and the difference is about four to five times. In addition, the frequency doubling of the blade decays with the increase of the frequency, and the pressure pulsation decay of the high-position blade is linear, and that of the lower-position blade is exponential. 


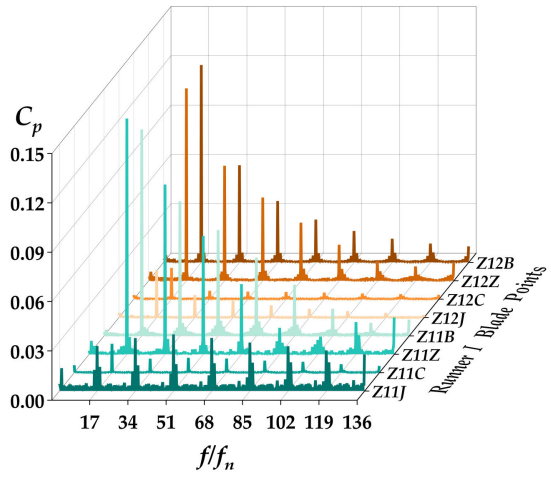

(a)

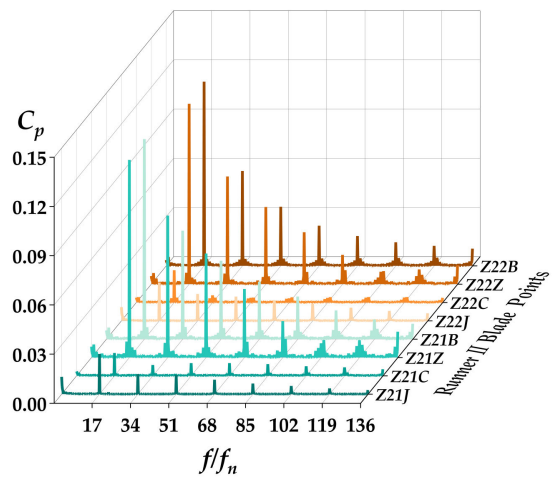

(b)

(A) OLP condition $\left(\mathrm{a}_{1}=142 \mathrm{~mm}\right)$

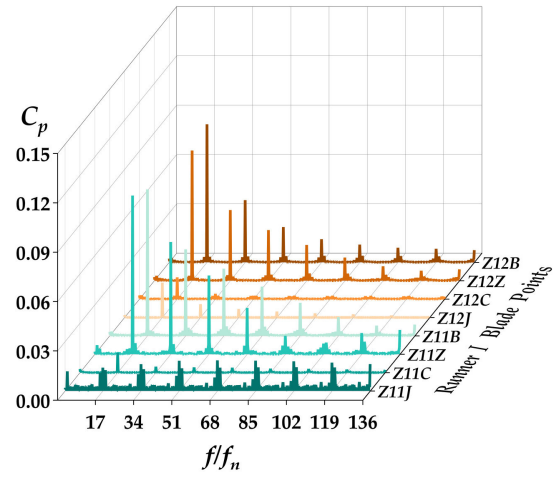

(a)

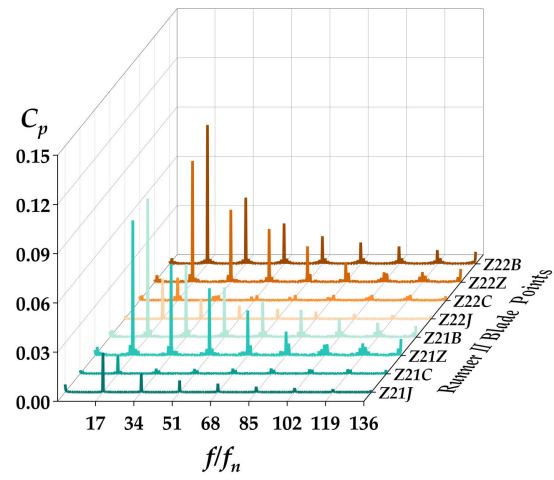

(b)

(B) BEP condition $\left(\mathrm{a}_{5}=110 \mathrm{~mm}\right)$

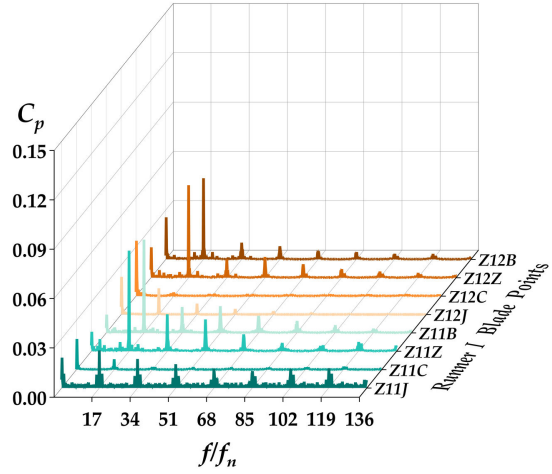

(a)

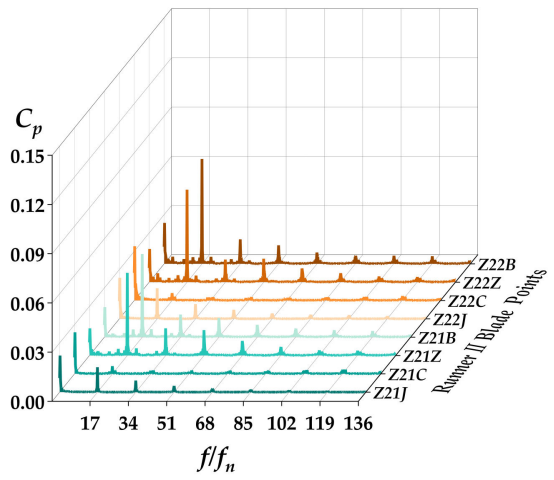

(b)

(C) PLP condition $(\mathrm{a} 8=77 \mathrm{~mm})$

Figure 19. Spectrum of pressure pulsation at runner blade monitoring points under different working conditions. (a) Data from blades of the runner I side; (b) data from blades of the runner II side.

The RBPF pressure pulsation and its frequency doubling is the normal pressure pulsation of the hydraulic turbine, which is associated with the operation of hydraulic turbine. The attenuation characteristics of the blade frequency doubling signal can reflect the flow regime in the turbine runner. As mentioned above, the amplitude of the blade frequency in the guide vane and runner attenuates with the decrease of the flow rate; therefore, the reasons for the difference of the frequency doubling attenuation law of the high-position blade and the low-position blade can be summarized as follows: Under the condition of the OLP and BEP, the mainstream at the outlet of the runner flows along the outer side of the collection elbow or the middle of the flow channel, and the flow rate on the outer side of the collection elbow is larger and more stable than that on the inner side, and the flow rate gradient is smaller than that on the inner side of the collection elbow. This state continues to affect the region, such as the runner and the guide vane. The runner blade is the place where the blade frequency and its doubling pressure pulsation originates from; therefore, the attenuation rate of the blade frequency and its doubling pressure pulsation on the surface of the high-position runner blade is lower than that on the low-position guide vane, showing a linear attenuation law.

\subsubsection{Analysis of Pressure Pulsation in Collection Elbow}

The collection elbow is one of the most complex parts of this double-runner turbine, where the water flow from the outlet of the runner converges and produces a large area of vortex, which not only affects the upstream runners and guide vanes, but also plays a decisive role in the development of water flow in the downstream draft tube. 
As can be seen from Figure 20, the low-frequency pressure pulsation is the main component of the pressure pulsation in the collection elbow, and there are very weak RBPF of $17 f n$ and GVPF of $20 f n$ signals under the condition of the OLP. This shows that the complex high-frequency components caused by rotor-stator interference basically attenuates and dissipates in the runner, and only the RBPF and the GVPF pressure pulsation are left in the draft tube. The low-frequency pressure pulsation component is an important characteristic of the flow in the collection elbow. Generally speaking, the low-frequency pressure pulsation is the strongest under the condition of the PLP, the local C $p$ reaches 0.06 , and the average $C p$ value is 0.02 , which is 10 times that of the $C p$ value that monitors at the BEP. For different monitoring points, at the OLP, the point with strong low-frequency pressure pulsation is located in the inner part of runner II and the outer part of runner I and the WSX2 of the outlet section of the collection elbow. At the BEP, the points with strong low-frequency pulsation are located at the inner side of the collection elbow and the monitoring points of WSX1 and WSX2 on the outlet section. At the PLP, the pressure pulsation is strong at the monitoring points near the confluence and the inner side of the elbow. The low-frequency pulsation is mainly related to the periodic movement of vortex flow and water vapor union in the draft tube. Based on the analysis of the distribution of pressure pulsation intensity and the flow field in this region, it is believed that the distribution of low-frequency pressure pulsation in the draft tube is quite random. In particular, under the condition of a low flow rate, vortices cluster in the draft tube, accompanied by the occurrence of cavitation, and the type of local water vapor union is complex, combined with the convergence and the spin motion of the two mainstreams from the outlet of two runners, where it is difficult to determine the location of the strong signal center of low-frequency pulsation intensity within a certain period of time. However, it is certain that at the dead-water zone where the vortex resides and the region where the flow fluctuates rapidly (such as the inner side of the collection elbow) it is easy to generate strong low-frequency pressure pulsation signals. Therefore, the arrangement of the draft tube should be able to avoid the occurrence of rapidly varied flows as far as possible when designing the flow force of the draft tube, which will effectively restrain the generation and development of pressure pulsation.

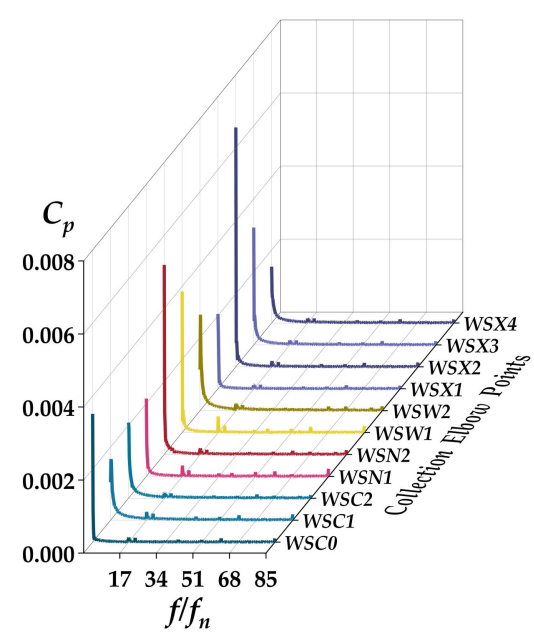

(A) OLP condition $\left(\mathrm{a}_{1}=142 \mathrm{~mm}\right)$

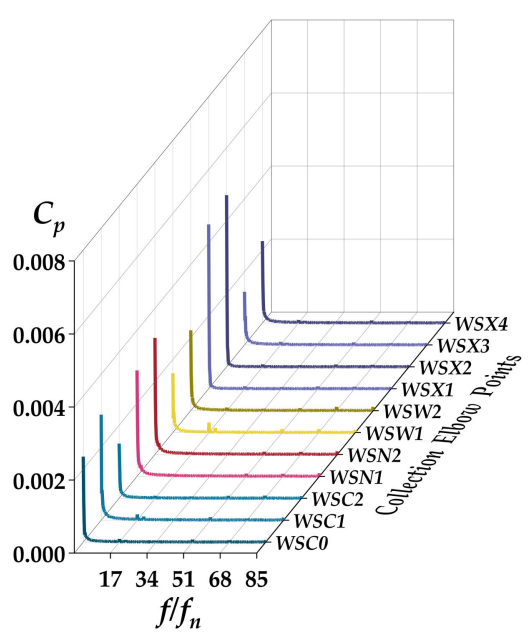

(B) BEP condition $\left(\mathrm{a}_{5}=110 \mathrm{~mm}\right)$

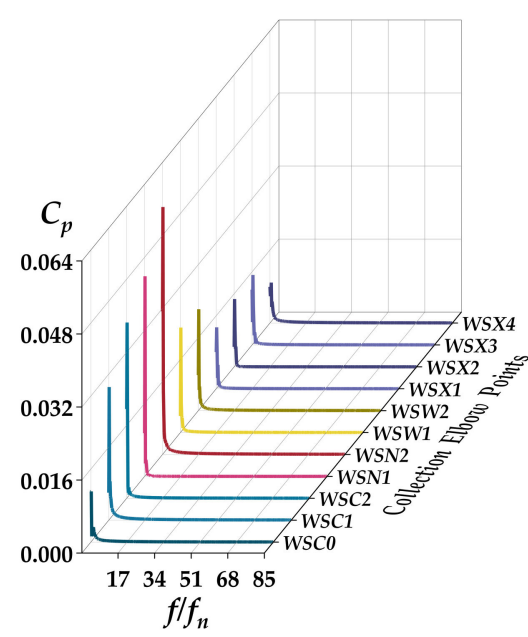

(C) PLP condition $(\mathrm{a} 8=77 \mathrm{~mm})$

Figure 20. Spectrum of pressure pulsation at the collection elbow monitoring point under different working conditions.

\section{Conclusions}

In this paper, the flow field of a horizontal axis double-runner Francis turbine was numerically simulated, and the variation laws between the flow rate of the two runners and the runner efficiency of the turbine were analyzed by using steady numerical calculation. 
Furthermore, an unsteady numerical calculation was used to analyze the variation law of the flow field in the turbine and the distribution and migration law of the pressure pulsation under different operating conditions. The results are as follows:

(1) When the guide vane opening is $a_{5}=110 \mathrm{~mm}$, the efficiency of the two runners is the highest, among which the efficiency of runner I is $93.23 \%$ and that of runner II is $92.78 \%$. The relationship between the average efficiency of the two runners and the flow rate difference is a negative correlation. The larger the flow rate difference between two runners, the lower the average efficiency of them. When the guide vane opening decreases, the runner power output drops more when the runner reaches maximum efficiency, which is shown by the deteriorated flow regime in the runner and the unbalanced and interfered flow of the two runners under a low flow rate. Therefore, we put forward two suggestions to improve the hydraulic performance. One is to improve the symmetry of the two runner channels as much as possible in the design stage, so as to maximize the average efficiency of the runners. Second, the actual operation should pay attention to adjust the flow rate of the two runners through the guide vane opening, and prepare to cut off the connection of a runner when the flow is lower than BEP.

(2) Due to the asymmetry of water flow at different heights caused by the vertical arrangement of the runners, the pressure on the surface of runner blades will change in a position-dependent distribution. The hydraulic turbine studied in this paper adopts the runner outlet arrangement with an opposite outflow, so the flow field inside the runner is mainly affected by the flow steering at the collection elbow. When the flow rate is different, the mainstream direction and convergence area in the elbow will change, forcing the stagnant area of the vortex to migrate in the draft tube cone, thus affecting the flow regime in the runner by backtracking upstream, and determining the formation and extinction of the vortex in the downstream draft tube, which will change the operation efficiency and stability of the machine under the interaction of the whole draft tube area. We believe that optimizing the collection elbow structure, such as reducing the curvature of the elbow or moving the flow convergence point, can bring reliable benefits to the improvement of the runners' flow regimes, and this work needs to be further carried out in future study.

(3) When the working conditions change, the pulsation area of low-frequency and strong pressure on the surface of the guide vanes will migrate regularly. As the flow rate decreases, the pulsation amplitude of low-frequency pressure will first decrease and then increase, and the strong signal area of the pressure pulsation on the surface of the guide vanes will move from the runner II side to the runner I side and change from the low-position guide vanes to the high-position one. Inside the runners, the attenuation of the pressure pulsation of the high-position blade accords with the linear law, and that of the low-position blade accords with the exponential law. The pressure pulsation in the draft tube is mainly a low-frequency one, and the distribution of pressure pulsation in the draft tube is quite random, but the low-frequency pressure pulsation signal is easy to be strengthened in the dead water zone where the vortex resides and near the region where the water flow changes rapidly. After obtaining the distribution and transmission law of pressure pulsation in the hydraulic turbine, we can take appropriate measures to prevent abnormal pressure pulsation, which was the motivation of this paper to explore and show the change law of pressure pulsation in large length. How to use these laws to improve the operation of the turbine is the next task to be carried out.

Author Contributions: Conceptualization, Y.Z. and C.Y.; methodology, D.X.; software, D.X.; validation, C.Y., D.X. and J.W.; formal analysis, C.Y. and Y.Z.; investigation, J.W.; resources, C.Y. and Y.Z.; data curation, X.H. and Z.L.; writing — original draft preparation, J.W.; writing-review and editing, J.W.; visualization, J.W.; supervision, X.H. and Z.L.; project administration, C.Y.; funding acquisition, C.Y. All authors have read and agreed to the published version of the manuscript. 
Funding: This research was funded by National Key Research and Development Project, grant number 2019YFE0105200 and Fundamental Research Funds for the Central Universities, grant number B210202060.

Data Availability Statement: Not applicable.

Acknowledgments: The authors would like to thank anonymous referees for their valuable comments in improving the overall quality of a preliminary version of this paper.

Conflicts of Interest: The authors declare no conflict of interest.

\section{References}

1. Elbatran, A.H.; Yaakob, O.B.; Ahmed, Y.M.; Shabara, H.M. Operation, performance and economic analysis of low head microhydropower turbines for rural and remote areas: A review. Renew. Sustain. Energy Rev. 2015, 43, 40-50. [CrossRef]

2. Tiwari, G.; Kumar, J.; Prasad, V.; Patel, V.K. Utility of CFD in the design and performance analysis of hydraulic turbines-A review. Energy Rep. 2020, 6, 2410-2429. [CrossRef]

3. Ding, K. Brief analysis of technical characteristics and application prospects of horizontal double runner francis hydro-generator units. Des. Hydropower Stn. 2017, 33, 10-13.

4. Cao, W.Y. Research and Application of Double Runner Hydrogenerator Unit. In Proceedings of the Zhongguo Shuili Xuehui 2008 Xueshu Nian Hui, Haikou, China, 28 October 2008; p. 3.

5. Robert, D.; Nesweb, J. For a large flow rate change double-runner Francis turbine. Mech. Electr. Tech. Hydropower Stn. 1996, 3 , 67-70.

6. Chen, S.W. Application of Double-Runner Hydrogenerator Unit in Hydropower Station. Small Hydro Power 2015, 5, 53-54. [CrossRef]

7. Robert, D.; Tai, C.L. Application of double runner Francis hydraulic turbine in large flow rate change. Guowai Dadianji 1995, 15, 75-78.

8. Zheng, Y.; Yang, C.X.; Zhou, D.Q.; Shen, M.H.; Li, X.X. Optimization design of horizontal Francis turbine with two runners. J. Drain. Irrig. Mach. Eng. 2012, 30, 341-345.

9. Yang, C.X.; Zheng, Y.; Zhou, D.Q. Optimization Design and Numerical Simulation of Horizontal Francis Turbine with Two Runners. In Proceedings of the The 4th Hydraulic Machinery \& System Conference of China, Lanzhou, China, 16-18 September 2011; pp. 377-381.

10. Benigni, H.; Schiffer, J.; Jaberg, H. Refurbishment of twin Francis turbines-Maximizing the annual production. IOP Conf. Ser. Earth Environ. Sci. 2019, 240, 022036. [CrossRef]

11. Ondrej, R.; Dalibor, C. Increasing the Efficiency of Induction Generator in Small Hydro Power Plant for Varying River Flow Rate; Springer International Publishing: Berlin/Heidelberg, Germany, 2018; pp. 169-176.

12. Yu, B.L.; Fang, D.B.; Meng, J.X. Analysis of the generation efficiency of disaggregated renewable energy and its spatial heterogeneity influencing factors: A case study of China. Energy 2021, 234, 121295. [CrossRef]

13. Qian, Z.D.; Zheng, B.; Huai, W.X.; Lee, Y.H. Analysis of pressure oscillations in a Francis hydraulic turbine with misaligned guide vanes. Proc. Inst. Mech. Eng. Part A J. Power Energy 2009, 224, 139-152. [CrossRef]

14. Song, H.; Pang, J.Y.; Wang, H.T. Influence of guide vane opening on pressure pulsation characteristics of a mixed-flow turbine. Gansu Water Resour. Hydropower Technol. 2021, 57, 65. [CrossRef]

15. Maiwald, M. Influence of Hydraulic Design on Stability and on Pressure Pulsations in Francis Turbines at Overload, Part Load and Deep Part Load based on Numerical Simulations and Experimental Model Test Results. IOP Conf. Ser. Earth Environ. Sci. 2014, 22, 032013. [CrossRef]

16. Nennemann, B.; Désy, N. Draft tube pressure pulsation predictions in Francis turbines with transient Computational Fluid Dynamics methodology. IOP Conf. Ser. Earth Environ. Sci. 2014, 22, 032002. [CrossRef]

17. Minakov, A.V.; Platonov, D.V.; Dekterev, A.A.; Sentyabov, A.V.; Zakharov, A.V. The analysis of unsteady flow structure and low frequency pressure pulsations in the high-head Francis turbines. Int. J. Heat Fluid Flow 2015, 53, 183-194. [CrossRef]

18. Minakov, A.V.; Platonov, D.V.; Dekterev, A.A.; Sentyabov, A.V.; Zakharov, A.V. The numerical simulation of low frequency pressure pulsations in the high-head Francis turbine. Comput. Fluids 2015, 111, 197-205. [CrossRef]

19. Trivedi, C.; Gogstad, P.J.; Dahlhaug, O.G. Investigation of the unsteady pressure pulsations in the prototype Francis turbines-Part 1: Steady state operating conditions. Mech. Syst. Signal Process. 2018, 108, 188-202. [CrossRef]

20. Favrel, A.; Lee, N.; Irie, T.; Miyagawa, K. Design of Experiments Applied to Francis Turbine Draft Tube to Minimize Pressure Pulsations and Energy Losses in Off-Design Conditions. Energies 2021, 14, 3894. [CrossRef]

21. Wu, Y.L.; Liu, S.H.; Dou, H.S.; Wu, S.F.; Chen, T.J. Numerical prediction and similarity study of pressure fluctuation in a prototype Kaplan turbine and the model turbine. Comput. Fluids 2012, 56, 128-142. [CrossRef]

22. Zuo, Z.G.; Liu, S.H.; Liu, D.M.; Qin, D.Q.; Wu, Y.L. Numerical analyses of pressure fluctuations induced by interblade vortices in a model Francis turbine. J. Hydrodyn. Ser. B 2015, 27, 513-521. [CrossRef]

23. Liu, S.H.; Shao, Q.; Yang, J.M.; Wu, Y.L.; Dai, J. Unsteady turbulent simulation of prototype hydraulic turbine and analysis of pressure fluctuation in draft tube. J. Hydroelectr. Eng. 2005, 24, 74-78. 
24. Feng, J.J.; Wu, H.; Wu, G.K.; Guo, P.C.; Luo, X.Q. Numerical simulation of pressure fluctuation in a Francis turbine at part load conditions with improved measures. J. Hydraul. Eng. 2014, 45, 1099-1105. [CrossRef]

25. Ji, B.; Luo, X.W.; Nishi, M.; Xu, H.Y. Analysis on characteristics of two-stage rotor-stator interaction and pressure fluctuation propagation in Francis turbine under partial load. J. Hydroelectr. Eng. 2014, 33, 191-196.

26. Zheng, Y.; Jiang, W.Q.; Chen, Y.J.; Sun, A. Investigation on Low Frequency Pulsating and Draft Tube Vortex of Tubular Turbine. Trans. Chin. Soc. Agric. Mach. 2018, 49, 165-171.

27. Su, W.T.; Binama, M.; Li, Y.; Zhao, Y. Study on the method of reducing the pressure fluctuation of hydraulic turbine by optimizing the draft tube pressure distribution. Renew. Energy 2020, 162, 550-560. [CrossRef]

28. Xie, T.T.; Wang, T.T.; Cao, W.Z.; Zhang, C.B. Research on Hydraulic Vibration and Energy Loss of Multi-turbine System in Hydropower Station. Water Power 2021, 47, 88-91.

29. Yang, M.J.; Chen, D.; Zhang, C.B.; Li, J.Y. Analysis of Pressure Pulsation and Hydraulic Vibration for Multiple Francis Turbines. China Rural Water Hydropower 2018, 3, 166-170, 175. [CrossRef]

30. Li, W.; Qian, Z.D.; Gao, Y.Y. Comparison of pressure oscillation characteristics in a Francis hydraulic turbine with four different turbulence models. Eng. J. Wuhan Univ. 2013, 46, 174-179.

31. Qian, Z.D.; Yang, J.D. Comparison of numerical simulation of pressure pulsation in Francis hydraulic turbine by different turbulence models. J. Hydroelectr. Eng. 2007, 26, 111-115.

32. Ondrej, R.; Dalibor, C. Efficiency optimization of small hydro power plant in low power operation. In Proceedings of the 2017 IEEE International Conference on Environment and Electrical Engineering and 2017 IEEE Industrial and Commercial Power Systems Europe (EEEIC/I\&CPS Europe), Milan, Italy, 6-9 June 2017; pp. 1-5.

33. Xu, X.P.; Zhou, Z. Application of Polyhedral Mesh in CFD. Flight Dyn. 2009, 6, 87-89, 93.

34. Wei, C.Z.; Li, Y.H.; Yi, H. Application of Polyhedral Mesh in Numerical Simulations of Flow Around Hulls. J. Shanghai Jiao Tong Univ. 2016, 50, 1144-1151. [CrossRef]

35. Yu, A.; Tang, Q.H.; Wang, X.C.; Zhou, D.Q.; Liu, J.T. Investigation of the Pressure Fluctuation Alleviation in a Hydraulic Turbine by Runner Modification. Water 2019, 11, 1332. [CrossRef]

36. Shih, T.H.; Liou, W.W.; Shabbir, A.; Yang, Z.G.; Zhu, J. A new k- $\epsilon$ eddy viscosity model for high reynolds number turbulent flows. Comput. Fluids 1995, 24, 227-238. [CrossRef]

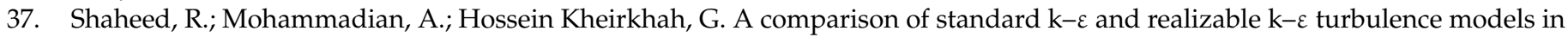
curved and confluent channels. Environ. Fluid Mech. 2019, 19, 543-568. [CrossRef]

38. Guo, P.C.; Sun, L.G.; Luo, X.Q. Flow characteristic investigation into inter-blade vortex for Francis turbine. Trans. Chin. Soc. Agric. Eng. 2019, 35, 43-51. [CrossRef] 